\title{
The substrate tolerance of alcohol oxidases
}

\author{
Mathias Pickl $^{2}$ - Michael Fuchs ${ }^{2}$ - Silvia M. Glueck ${ }^{1,2}$ • \\ Kurt Faber $^{2}$
}

Received: 7 April 2015 /Revised: 10 May 2015 / Accepted: 15 May 2015 /Published online: 8 July 2015

(C) The Author(s) 2015. This article is published with open access at Springerlink.com

\begin{abstract}
Alcohols are a rich source of compounds from renewable sources, but they have to be activated in order to allow the modification of their carbon backbone. The latter can be achieved via oxidation to the corresponding aldehydes or ketones. As an alternative to (thermodynamically disfavoured) nicotinamide-dependent alcohol dehydrogenases, alcohol oxidases make use of molecular oxygen but their application is under-represented in synthetic biotransformations. In this review, the mechanism of copper-containing and flavoprotein alcohol oxidases is discussed in view of their ability to accept electronically activated or non-activated alcohols and their propensity towards over-oxidation of aldehydes yielding carboxylic acids. In order to facilitate the selection of the optimal enzyme for a given biocatalytic application, the substrate tolerance of alcohol oxidases is compiled and discussed: Substrates are classified into groups (non-activated prim- and sec-alcohols; activated allylic, cinnamic and benzylic alcohols; hydroxy acids; sugar alcohols; nucleotide alcohols; sterols) together with suitable alcohol oxidases, their microbial source, relative activities and (stereo)selectivities.
\end{abstract}

Keywords Oxidation - Biocatalysis · Alcohol oxidase · Substrate tolerance $\cdot$ Flavoprotein $\cdot \mathrm{Cu}$-containing oxidase

Electronic supplementary material The online version of this article (doi:10.1007/s00253-015-6699-6) contains supplementary material, which is available to authorized users.

Kurt Faber

kurt.faber@uni-graz.at

1 Austrian Centre of Industrial Biotechnology (ACIB GmbH), Petersgasse 14, 8010 Graz, Austria

2 Department of Chemistry, Organic \& Bioorganic Chemistry, University of Graz, Heinrichstrasse 28, A-8010 Graz, Austria

\section{Introduction}

Oxidation represents a fundamental reaction in nature (Hollmann et al. 2011; Turner 2011), and oxidases are a prominent subclass of redox enzymes, which use oxygen either as oxidant or as electron acceptor. This property made them particularly attractive for the production of chemicals (Vennestrom et al. 2010). In this context, the oxidation of alcohols is an important transformation in synthetic chemistry, which allows to introduce carbonyl groups, which represent excellent acceptors for $\mathrm{C}$-, $\mathrm{N}$-, $\mathrm{O}$ - and $\mathrm{S}$-nucleophiles and thereby allows the extension of a given carbon backbone. Consequently, a large number of protocols has been developed, which depend on (i) transition metals in stoichiometric (e.g. Cr, Mn) or catalytic amounts (e.g. Ru, Fe), (ii) metal-free oxidations according to Swern or Pfitzner-Moffat (Pfitzner and Moffatt 1963; Omura and Swern 1978), (iii) molecular oxygen as oxidant (Tojo and Fernández 2006) and more recently, (iv) organocatalysts, such as TEMPO (Wertz and Studer 2013).

In a related fashion, alcohol oxidases convert primary and secondary alcohols to aldehydes and ketones, respectively. During this reaction, molecular oxygen is reduced to hydrogen peroxide. In order to avoid enzyme deactivation, a catalase is usually employed, particularly on preparative scale. For screening purposes, a spectrophotometric assay based on horse radish peroxidase (HRP) together with a suitable artificial electron acceptor, such as 2,2'-azino-bis(3ethylbenzthiazoline-6-sulfonic acid) (ABTS) may be employed (Scheme 1). The ABTS-radical generated shifts its absorption maximum (Baron et al. 1994; Uwajima and Terada 1980).

Although cofactor-lacking oxidases are reported (Fetzner and Steiner 2010), commonly used alcohol oxidases depend on flavin (Macheroux et al. 2011; Dijkman et al. 2013) or a 


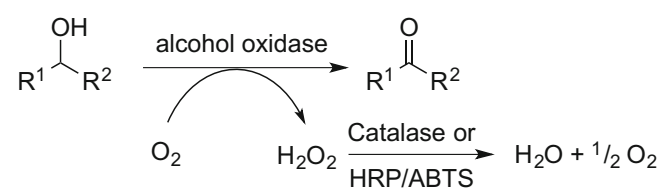

Scheme 1 Biocatalytic oxidation of alcohols using an alcohol oxidase

metal $(\mathrm{Cu})$ as a cofactor (Whittaker 2003), which mediates the electron transfer. In flavoprotein oxidases, the oxidation proceeds via two half reactions, where the alcohol is first oxidised by a two-electron transfer during the reductive half reaction, yielding reduced flavin. The oxidised flavin is regenerated by a stepwise single-electron transfer via the oxidative half reaction, which requires triplet oxygen, as it is a spinforbidden reaction. Hence, di-oxygen acts as single-electron acceptor and forms superoxide $\left(\mathrm{O}_{2}^{-}\right)$, stabilised by a positively charged histidine residue (Dijkman et al. 2013; Wongnate et al. 2014). Another single-electron transfer yields a covalent hydroperoxy flavin intermediate, which eliminates hydrogen peroxide and re-forms oxidised flavin (Scheme 2) (Gadda 2012). The highly unstable $\mathrm{C}_{4} \mathrm{a}$-hydroperoxyflavin intermediate has only been detected for pyranose oxidase (P2O) (Mattevi 2006; Chaiyen et al. 2012; Wongnate and Chaiyen 2013).

The oxidation of primary alcohols catalysed by flavoprotein oxidases does not necessarily stop at the aldehyde stage, but may further proceed to the corresponding carboxylic acid. This second oxidation step is mechanistically less investigated, but it is obvious that the actual substrate is the aldehyde hydrate (gem-diol), rather than its carbonyl form, because hydride abstraction from the former yields a doubly resonance- stabilised oxocarbenium cation, which upon expulsion of $\mathrm{H}^{+}$ furnishes the carboxylic acid. In contrast, hydride abstraction from the carbonyl form would lead to a highly unstable (hypothetical) acylium cation, which would be quenched by a water molecule (Scheme 3).

This mechanism for over-oxidation has been proposed for choline oxidase (CHO), whose natural role is the formation of $N$-trimethylammonium glycine ('betaine') from choline via the aldehyde hydrate through a two-step oxidation (Scheme 4) (Rungsrisuriyachai and Gadda 2008).

The over-oxidation of alcohols to carboxylic acids has been observed not only for choline oxidase but also for other flavoprotein oxidases, such as alditol oxidase (AldO), aryl alcohol oxidase (AAO), hydroxymethyl furfuryl oxidase (HMFO), hexose oxidase (HOX, Dbv29), isoamyl alcohol oxidase (IAO) or short- and long-chain alcohol oxidases (SCAOs, LCAOs). Labelling studies proved the existence of the aldehyde hydrate as intermediate (Van Hellemond et al. 2009), and for AAO, which naturally oxidises benzylic alcohols, NMR studies revealed that the gem-diol intermediate was favoured (Ferreira et al. 2010) (Scheme 3).

Structurally, most of the flavoprotein oxidases either belong to the glucose-methanol-choline (GMC) oxidase or the vanillyl alcohol oxidase (VAO) family. Both families have a flavin present in the active site where the binding domain and the binding mode of the flavin differ. In case of VAO, the flavin is covalently linked to a histidine, cysteine or tyrosine residue, while in the GMC family, the majority of the enzymes contain a dissociable flavin adenine dinucleotide (FAD) moiety. In $\mathrm{P} 2 \mathrm{O}$ or $\mathrm{CHO}$, a covalent linkage was found. The active sites and consequently the substrate scope of these enzymes
Scheme 2 Catalytic cycle of flavin-containing alcohol oxidases

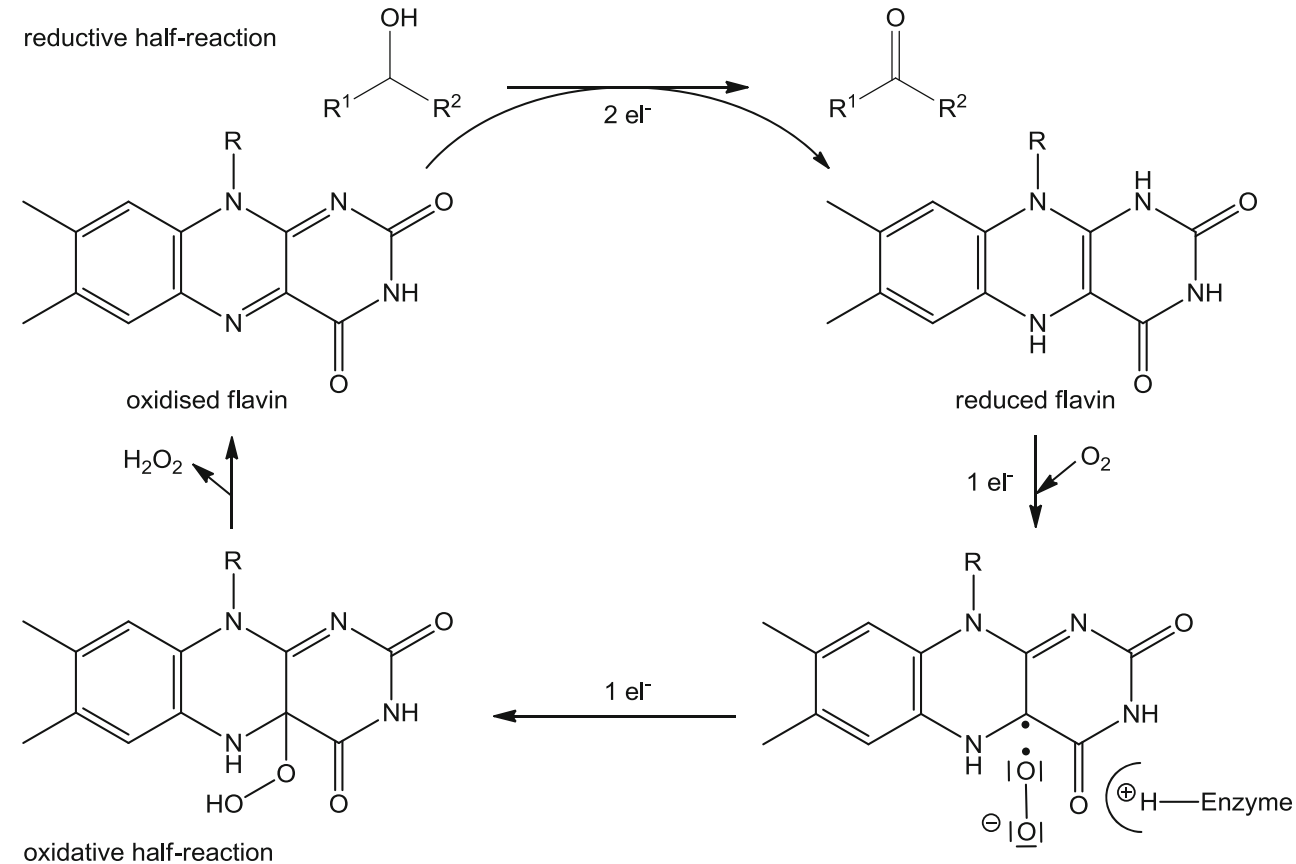




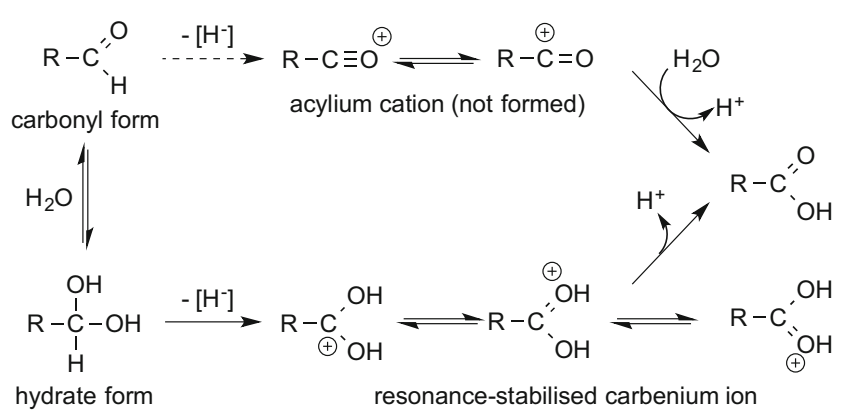

Scheme 3 Aldehyde oxidation via the hydrate intermediate

show high variance (Fraaije et al. 1998a; Kiess et al. 1998; Leferink et al. 2008; Dijkman et al. 2013).

Another redox cofactor found in alcohol oxidases, such as galactose oxidase (GOase), is the transition metal copper, whose role in catalysis is well described in several reviews (Ridge et al. 2008; Guengerich 2013). Since only a single copper(I) ion is found in the active site, it seems surprising that a two-electron transfer can occur. Detailed investigations revealed that the latter proceeds via two consecutive singleelectron transfer steps. Thus, abstraction of the first electron by $\mathrm{Cu}^{2+}$ yields $\mathrm{Cu}^{+}$, which transfers its electron onto a tyrosine residue, which forms a transient radical anion (Monti et al. 2011). The latter is stabilised by a rare covalent thioether bridge with an adjacent cysteine (Ito et al. 1991). The second electron transfer yields a $\mathrm{Cu}^{+}$-tyrosine radical. From this, oxygen accepts two electrons (Wang 1998; Whittaker 2003) (Scheme 5). GOase from Fusarium NRRL 2903 is the most prominent member of $\mathrm{Cu}$-containing alcohol oxidases and belongs to the family of radical copper oxidases, a family with a wide phylogenetic distribution and broad range of functions. The crystal structure of the enzyme revealed that a mononuclear copper ion is centred in a distorted pyramid structure, which is coordinated by two tyrosine residues (Tyr272 and Tyr495) and two histidine side chains (His496 and His581) (Whittaker and Whittaker 2001).

For $\mathrm{Cu}$-containing alcohol oxidases, the oxidation stops at the aldehyde stage and over-oxidation was not observed (Monti et al. 2011).

From a biocatalytic viewpoint, alcohol oxidases are a promising group of enzymes, because they are biochemically well characterised and a broad range of enzymes have been described (Whittaker 2003; Leferink et al. 2008; Dijkman et al. 2013) which were also employed in cascade reactions (Fuchs et al. 2012; Perez-Sanchez et al. 2013; Schrittwieser et al. 2011). Depending on their role in nature, substrates for alcohol oxidases vary to a great extent in terms of substrate

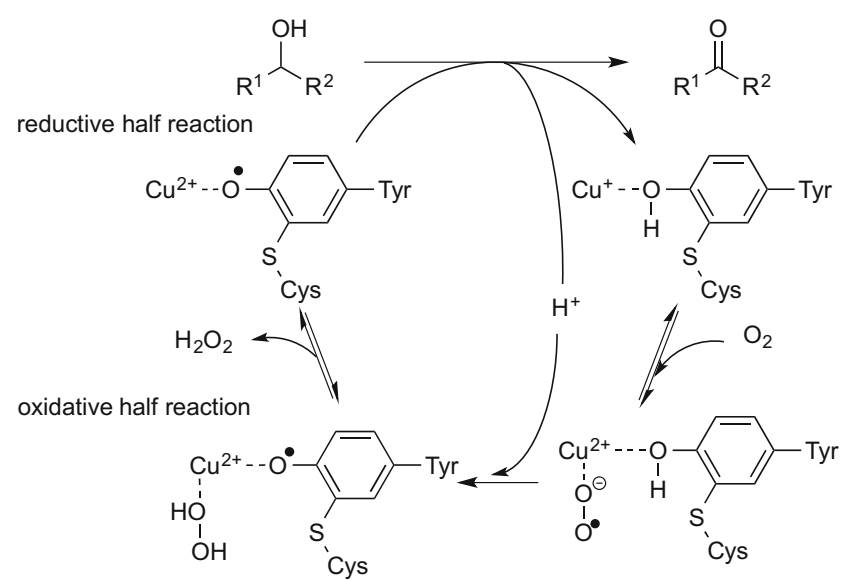

Scheme 5 Catalytic cycle of copper-containing oxidases

size and/or polarity (Turner 2011). In fungi, extracellular alcohol oxidases produce hydrogen peroxide (needed for lignin degradation by peroxidases) by oxidation of cinnamyl alcohols (e.g. coniferyl, coumaryl and sinapyl alcohol). Furthermore, hydrogen peroxide acts as antibiotic in the rhizosphere to protect roots (Monti et al. 2011). As an alternative to alcohol oxidases, $\mathrm{NAD}(\mathrm{P})^{+}$-dependent alcohol dehydrogenases provide a well-investigated enzyme platform for the oxidation of prim- and sec-alcohol functionalities. Although these enzymes are more abundant than alcohol oxidases, the equilibrium for oxidation is strongly disfavored but can be overcome by $\mathrm{NAD}(\mathrm{P})^{+}$recycling (Hollmann et al. 2011).

In the following, an overview on the current literature of alcohol oxidases is given, by focussing on their substrate tolerance to facilitate the choice of an appropriate enzyme for a given type of alcohol substrate.

\section{Non-activated alcohols}

\section{Primary aliphatic alcohols}

The enzymatic oxidation of non-activated aliphatic prim-alcohols by alcohol oxidases shows a remarkably broad substrate tolerance (Table 1) and encompasses straight-chain or branched substrates with chain lengths ranging from $\mathrm{C}_{1}$ to $\mathrm{C}_{16}$. In addition, functional groups, such as aromatics, halogens $(\mathrm{Cl}, \mathrm{Br})$, non-allylic olefins, alkylamino groups and carboxylates, are tolerated (Scheme 6). Vicinal, 1,3- and $\alpha, \omega$ diols are selectively oxidised at the prim-hydroxy group, while sec-alcohols remain untouched. Depending on the
Scheme 4 Two-step oxidation of choline by choline oxidase yielding betaine

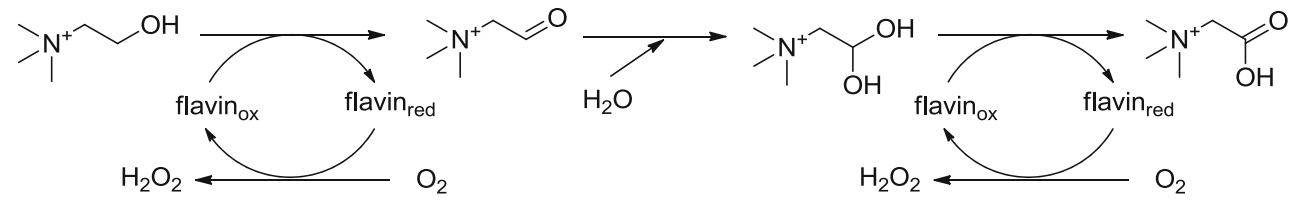


促

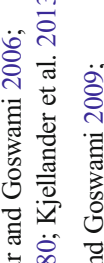

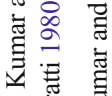

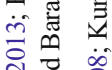

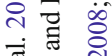

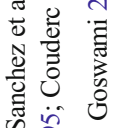

离

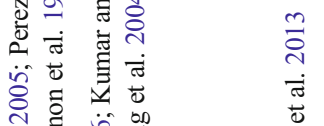

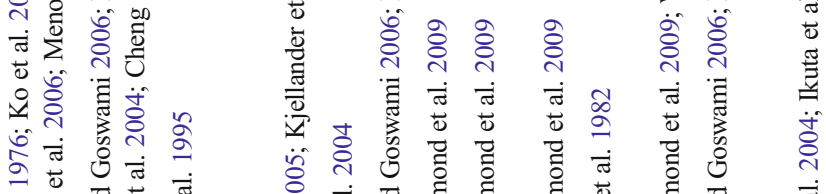

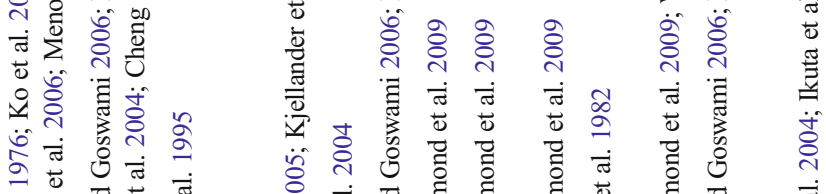

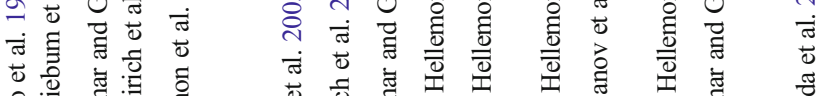

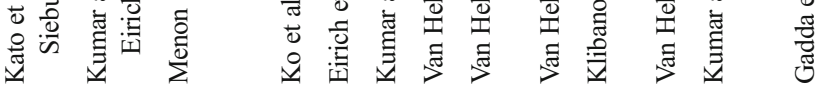

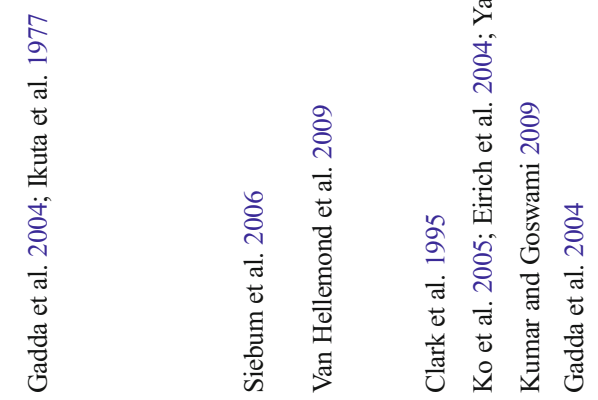

:

نे

î

บ

का एं

हิ

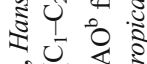

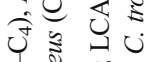

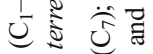

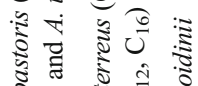

造

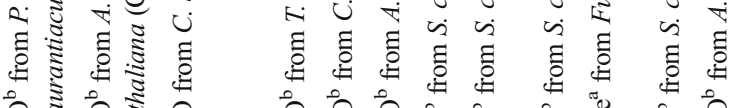

出

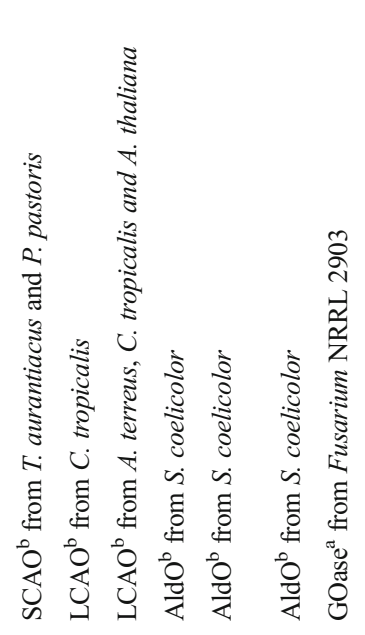

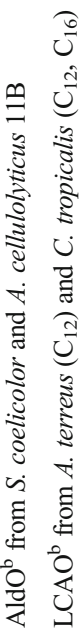

בิ
5
0
0
00
1
0
0
0
0
0
0
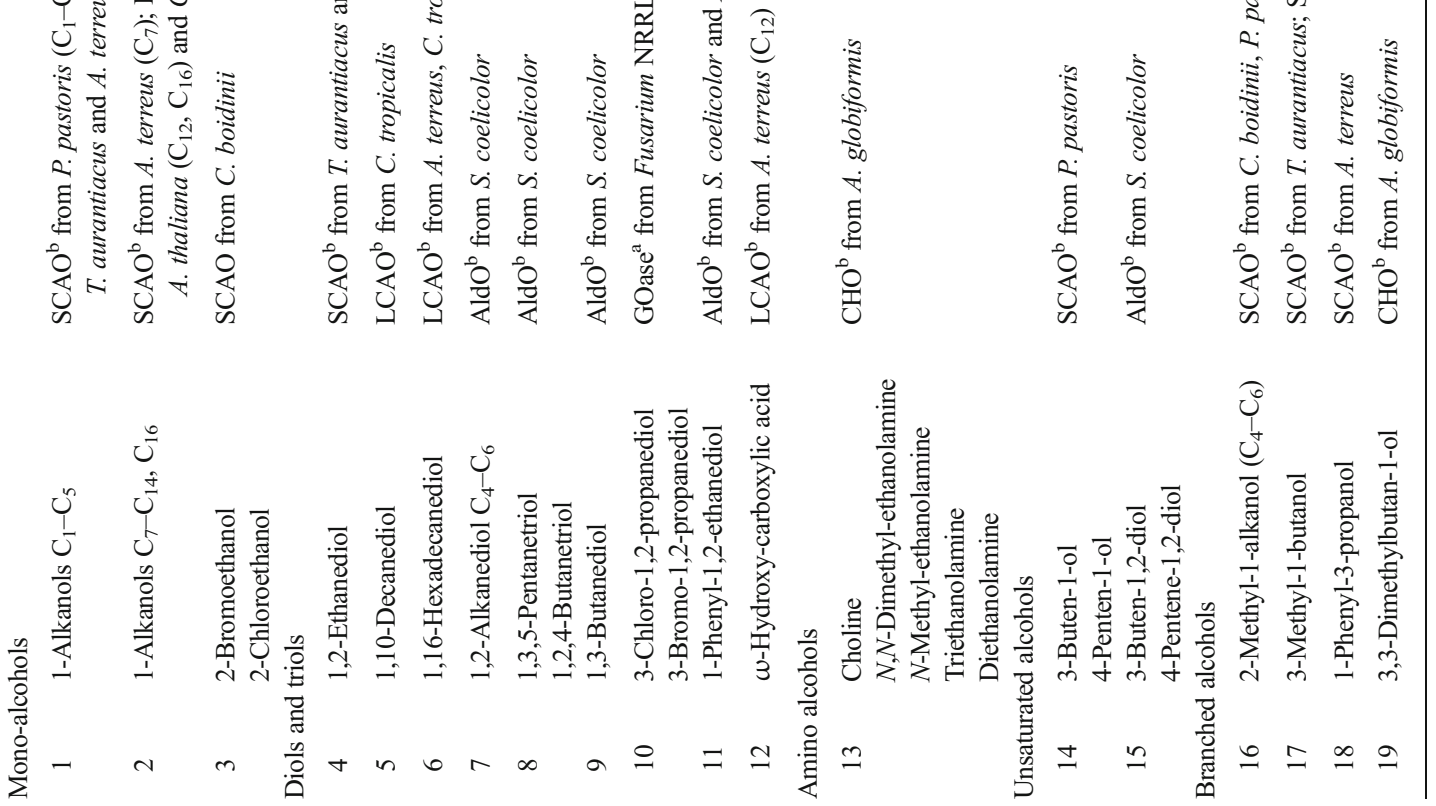


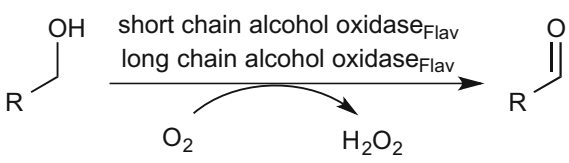

$\mathrm{R}=$ alkyl (short and long), $\omega$-hydroxyalkyl, $\omega$-haloalkyl, carboxyalkyl, 2- or 3-methylalkyl, $\omega$-alkenyl, 3-phenylalkyl

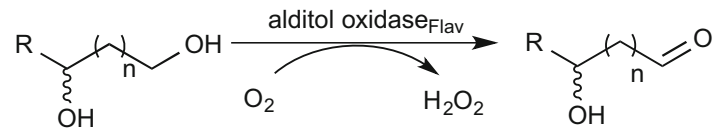

$\mathrm{n}=0, \mathrm{R}=$ alkyl, hydroxyalkyl, alkenyl, aryl

$\mathrm{n}=1, \mathrm{R}=$ alkyl, hydroxyalkyl

Scheme 6 Oxidation of primary alcohols by oxidases

enzyme, the oxidation products are the corresponding aldehydes and carboxylic acids, which are formed by overoxidation with flavoprotein oxidases.

Aliphatic alcohols with a chain length of one to seven C atoms were oxidised by short-chain alcohol oxidases (SCAOs) [EC 1.1.3.13] from Pichia pastoris, Hansenula sp. (Table 1, entry 1) and Aspergillus terreus (Table 1, entry 2) while methanol and ethanol were also converted by alcohol oxidases from Candida boidinii, Thermoascus aurantiacus and A. terreus (Table 1, entry 1) (Kato et al. 1976; Siebum et al. 2006; Menon et al. 1995; Couderc and Baratti 1980; Kumar and Goswami 2006; Ko et al. 2005; Perez-Sanchez et al. 2013). In general, the activity decreases with increasing chain length of the fatty alcohol, e.g. 1-pentanol shows $24 \%$ relative activity compared to methanol (Ko et al. 2005). SCAO from Hansenula sp. was employed together with a $\mathrm{C}-\mathrm{C}$ lyase in a cascade reaction, where short-chain alcohols (methanol, ethanol, 1-propanol and 1-pentanol) were oxidised with excellent conversion to the corresponding aldehydes, which were subjected to cross-acyloin condensation with benzoin generated in situ from benzaldehyde by benzaldehyde lyase to yield 2-hydroxyketones (Shanmuganathan et al. 2012; Perez-Sanchez et al. 2013). prim-Alcohols with a chain length of 7 to 16 carbon atoms were best oxidised by longchain alcohol oxidases (LCAOs) [EC 1.1.3.20] from A. terreus, Candida tropicalis and Arabidopsis thaliana (Table 1, entry 2). Both, SCAOs and LCAOs, are flavoproteins located in fungal microsomes (Kemp et al. 1988; Eirich et al. 2004; Kumar and Goswami 2006, Cheng et al. 2004). Terminal alcohols bearing a polar functional group, such as $\alpha$, $\omega$-diols (Table 1 , entries 5 and 6 ) and $\omega$-carboxy fatty alcohols (Table 1, entry 12), with a long hydrocarbon backbone were also oxidised by long-chain alcohol oxidases (Kumar and Goswami 2006).

Short-chain alcohol oxidase from several microorganisms (C. boidinii, Hansenula sp., P. pastoris and T. aurantiacus) was described to convert racemic branched alcohols (Table 1, entries 16-17) in an enantioselective fashion with conversions of $16-76 \%$, the non-reacted substrate enantiomers showed

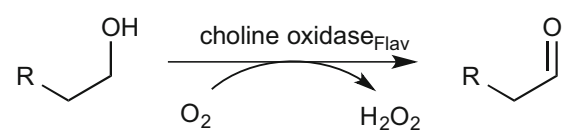

$\mathrm{R}=\mathrm{NMe}_{3}, \mathrm{NMe}_{2}, \mathrm{NHMe}$, $\mathrm{N}\left(\mathrm{CH}_{2} \mathrm{CH}_{2} \mathrm{OH}\right)_{2}$, $\mathrm{NHCH}_{2} \mathrm{CH}_{2} \mathrm{OH}, t-\mathrm{Bu}$

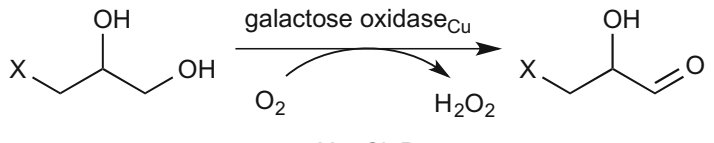

$\mathrm{X}=\mathrm{Cl}, \mathrm{Br}$

ees of up to $90 \%$ for SCAO from C. boidinii (Clark et al. 1995). Isoamyl alcohol oxidase (IAO) [EC 1.1.3.x] from $A s-$ pergillus oryzae exhibits a narrow substrate range and prefers branched short-chain alcohols, such as 3-methyl-1-butanol (Table 1, entry 17) (Yamashita et al. 1999). Halogensubstituted alcohols, which were oxidised by SCAO, were used as molecular probes for mechanistic studies (Menon et al. 1995).

Saturated and unsaturated vic-1,2-diols were the substrates of choice for alditol oxidase [EC 1.1.3.41] from Streptomyces coelicolor and Acidothermus cellulolyticus (Table 1, entries $7-9,11$ and 15). This enzyme apparently prefers a glycol or 1,3-diol moiety. For rac-1-phenyl-1,2-ethanediol carrying a bulky aryl moiety, the $(R)$-enantiomer was preferentially oxidised by alditol oxidase (Table 1, entry 11) (Van Hellemond et al. 2009). Short (non-allylic) unsaturated alcohols lacking a second hydroxy group were completely (4penten-1-ol) or partially (3-buten-1-ol) oxidised by shortchain alcohol oxidase from P. pastoris (Table 1, entry 14) (Siebum et al. 2006).

Another prominent enzyme of this group is choline oxidase from A. globiformis which oxidises choline and analogues, such as $N, N$-dimethylethanolamine, $N$-methylethanolamine, triethanolamine, diethanolamine and 3,3-dimethylbutan-1-ol (Table 1, entries 13 and 19) in a two-step oxidation to the corresponding carboxylic acid (Ikuta et al. 1977; Gadda et al. 2004).

\section{Secondary aliphatic alcohols}

Racemic secondary aliphatic alcohols are interesting substrates, because enantioselectivities in kinetic resolution are usually much higher than with prim-alcohols bearing a stereogenic centre. In contrast to prim-alcohols, which may undergo over-oxidation to carboxylic acids, the oxidation products derived from sec-alcohols are solely ketones (Scheme 7). Compared to prim-alcohol oxidases, enzymes acting on secondary alcohols are less abundant, but several enzymes were found to be highly active (Table 2). Secondary alcohol oxidase (SAO) 


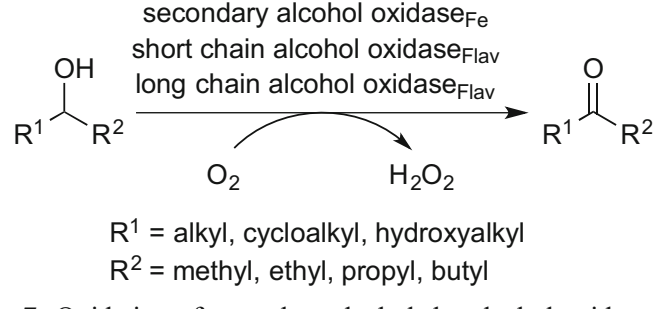

Scheme 7 Oxidation of secondary alcohols by alcohol oxidases

[EC 1.1.3.18] from Pseudomonas putida, Pseudomonas vesicularis and $A$. terreus has shown high activity for the polymeric substrate polyvinyl alcohol (PVA) (Table 2, entry 1) (Sakai et al. 1985; Kawagoshi and Fujita 1997), and it was discovered that one non-heme $\mathrm{Fe}^{2+}$ species is present in the enzyme. To date, it remains unclear whether the iron species serves as a cofactor like the copper in galactose oxidase or if it does not participate in catalysis at all.

For monomeric sec-alcohols, the relative activity of SAO from P. putida ranges between 5 and $30 \%$ (compared to PVA). High activity for 2-octanol was found with the enzyme from $P$. vesicularis ( $83 \%$ rel. activity), which also accepts cyclohexanol (42\% rel. activity). Its oxidised product (cyclohexanone) is used as a starting material for the synthesis of the polymer building block $\varepsilon$-caprolactam. sec-Alcohols bearing an additional $\mathrm{OH}$ group, such as 1,2-propanediol and 2,4-pentanediol, were also accepted as substrates (Table 2, entries 7 and 8); however, no details are reported about the regioselectivity of the oxidation (Sakai et al. 1985; Kawagoshi and Fujita 1997). Additionally, SCAO from T. aurantiacus, A. terreus and P. pastoris as well as LCAO from $C$. tropicalis showed broad activity on secondary alcohols (Table 2, entry 2) (Eirich et al. 2004; Kumar and Goswami 2009; Kjellander et al. 2013; Ko et al. 2005). Furthermore, 2-methyl-2-propanol was claimed to show $16 \%$ relative activity with SCAO, but this tert-alcohol should be a non-substrate (Ko et al. 2005).

\section{Activated alcohols}

\section{Allylic alcohols}

In contrast to saturated (non-activated) aliphatic alcohols, allylic and benzylic alcohols are much easier to oxidise, because radicals and carbene ions occurring as intermediates are resonance stabilised (see Electronic Supplementary Material, Scheme S1). Owing to their high intrinsic reactivity, allylic alcohols are easily oxidised by a broad range of alcohol oxidases, such as copper-containing galactose oxidase (GOase) [EC 1.1.3.9], flavoprotein cholesterol oxidase (ChOx) [EC 1.1.3.6], aryl alcohol oxidase (AAO) [EC 1.1.3.7] and hydroxymethylfurfural oxidase (HMFO) [EC 1.1.3.47] (Scheme 8, Table 3) (Guillen et al. 1992; Dieth et al. 1995; Sun et al. 2002; Dijkman and Fraaije 2014).

Small allylic alcohol was oxidised poorly by galactose oxidase (Table 3, entries 1 and 2), which prefers large analogues, such as cinnamyl alcohol (Table 3, entry 4). A mutant of galactose oxidase from Fusarium sp. oxidised cinnamyl alcohol with full conversion (Sun et al. 2002; Fuchs et al. 2012). In contrast to galactose oxidase, which does not accept sec-allylic alcohols, cholesterol oxidase from Rhodococcus erythropolis converted sterically demanding secondary allylic alcohols in a complete stereo- and enantioselective fashion with conversions up to $70 \%$ and high to excellent ees. For methyl-substituted bicyclic substrates (Table 3, entries 7 and 8), the relative (cis) position of the hydroxyl group with respect to the methyl group were mandatory to be accepted and

Table 2 Secondary aliphatic alcohols

\begin{tabular}{|c|c|c|c|}
\hline Entry & Substrate & Oxidase & Reference \\
\hline 1 & Polyvinyl alcohol & $\mathrm{SAO}^{\mathrm{a}}$ from P. putida and P. vesicularis & Sakai et al. 1985; Kawagoshi and Fujita 1997 \\
\hline 2 & 2-Alkanols $\mathrm{C}_{3}-\mathrm{C}_{12}, \mathrm{C}_{16}$ & $\begin{array}{l}\mathrm{SAO}^{\mathrm{a}} \text { from A. terreus }\left(\mathrm{C}_{3}, \mathrm{C}_{8}, \mathrm{C}_{12}\right) \text {, P. putida }\left(\mathrm{C}_{3}-\mathrm{C}_{7}\right) \\
\quad \text { and P. vesicularis }\left(\mathrm{C}_{4}-\mathrm{C}_{8}\right) ; \mathrm{SCAO}^{\mathrm{b}} \text { from T. aurantiacus } \\
\quad\left(\mathrm{C}_{3}-\mathrm{C}_{4}\right) \text {, A. terreus }\left(\mathrm{C}_{8}\right) \text { and P. pastoris }\left(\mathrm{C}_{3}\right) ; \mathrm{LCAO}^{\mathrm{b}} \\
\quad \text { from C. tropicalis }\left(\mathrm{C}_{10}-\mathrm{C}_{11}, \mathrm{C}_{16}\right)\end{array}$ & $\begin{array}{l}\text { Ko et al. 2005; Kumar and Goswami 2006; Kjellander et al. 2013; } \\
\text { Sakai et al. 1985; Kawagoshi and Fujita 1997; Kumar and } \\
\text { Goswami 2009; Eirich et al. } 2004\end{array}$ \\
\hline 3 & 3-Alkanols $\mathrm{C}_{5}-\mathrm{C}_{8}$ & $\begin{array}{l}\mathrm{SAO}^{\mathrm{a}} \text { from } P \text {. putida }\left(\mathrm{C}_{5}-\mathrm{C}_{8}\right) \text {, A. terreus }\left(\mathrm{C}_{8}\right) \\
\quad \text { and P. vesicularis }\left(\mathrm{C}_{6}-\mathrm{C}_{8}\right)\end{array}$ & Sakai et al. 1985; Kawagoshi and Fujita 1997; Eirich et al. 2004 \\
\hline 4 & 4-Alkanols $\mathrm{C}_{7}-\mathrm{C}_{10}$ & $\mathrm{SAO}^{\mathrm{a}}$ from $P$. putida $\left(\mathrm{C}_{7}-\mathrm{C}_{9}\right)$ and $P$. vesicularis $\left(\mathrm{C}_{7}, \mathrm{C}_{10}\right)$ & Sakai et al. 1985; Kawagoshi and Fujita 1997 \\
\hline 5 & 5-Nonanol & $\mathrm{SAO}^{\mathrm{a}}$ from $P$. putida & Sakai et al. 1985 \\
\hline 6 & Cycloalkanols $\mathrm{C}_{6}, \mathrm{C}_{8}$ & $\mathrm{SAO}^{\mathrm{a}}$ from $P$. vesicularis $\left(\mathrm{C}_{6}\right)$ and $A$. terreus $\left(\mathrm{C}_{8}\right)$ & Kawagoshi and Fujita 1997; Kumar and Goswami 2006 \\
\hline 7 & 1,2-Propanediol & $\mathrm{SAO}^{\mathrm{a}}$ from P. putida & Sakai et al. 1985 \\
\hline 8 & 2,4-Pentanediol & $\mathrm{SAO}^{\mathrm{a}}$ from $P$. vesicularis & Kawagoshi and Fujita 1997 \\
\hline
\end{tabular}

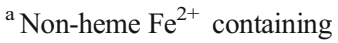

${ }^{\mathrm{b}}$ Flavin-containing 
galactose oxidase $\mathrm{Cu}_{\mathrm{C}}$ aryl alcohol oxidase Flav<smiles>[R]C=CCO</smiles>
cholesterol oxidase Flav $_{\text {flav }}$ hydroxymethylfurfural oxidase Flav $_{\text {}}$

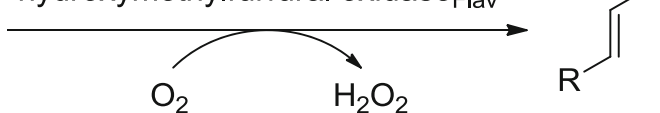

$\mathrm{R}=$ cycloalkyl, aryl

Scheme 8 Oxidation of allylic alcohols by alcohol oxidases

non-activated (non-allylic) hydroxy groups were unreactive. Even comparably small monocyclic substrates could be converted (Dieth et al. 1995). Aryl alcohol oxidase exhibits a broad substrate scope and accepts phenyl substituted allylic alcohols such as coniferyl and cinnamyl alcohol (Table 3, entries 4 and 5), as well as slim counterparts, such as 2,4hexadien-1-ol (Table 3, entry 3), which shows that this enzyme does not necessarily need a cyclic structure, but only a conjugated system (Ferreira et al. 2005; Romero et al. 2009). 5-Hydroxymethylfurfural oxidase exhibited a similar behaviour and appears to be a promising candidate for the oxidation of allylic alcohols, as it showed excellent acceptance of cinnamyl alcohol (Table 3, entry 4) and 2,4-hexadien-1-ol (Table 3, entry 3) (Dijkman and Fraaije 2014). With cinnamyl alcohol and its $p$-methoxy derivative, AAO shows overoxidation and forms the corresponding acids (Table 3, entry 4) (Guillen et al. 1992).

\section{Benzylic alcohols}

Due to their high degree of electronic activation, benzylic alcohols (Scheme 9, Table 4) are easy to oxidise. In particular, galactose oxidase from Fusarium NRRL 2903 and aryl alcohol oxidase from Pleurotus eryngii are ideally suited for this

Table 3 prim- and sec-Allylic alcohols

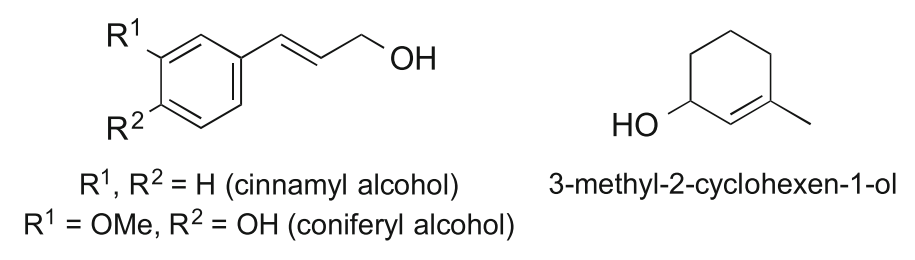

$\mathrm{R}^{1}, \mathrm{R}^{2}=\mathrm{H}$ (cinnamyl alcohol)

$\mathrm{R}^{1}=\mathrm{OMe}, \mathrm{R}^{2}=\mathrm{OH}$ (coniferyl alcohol)

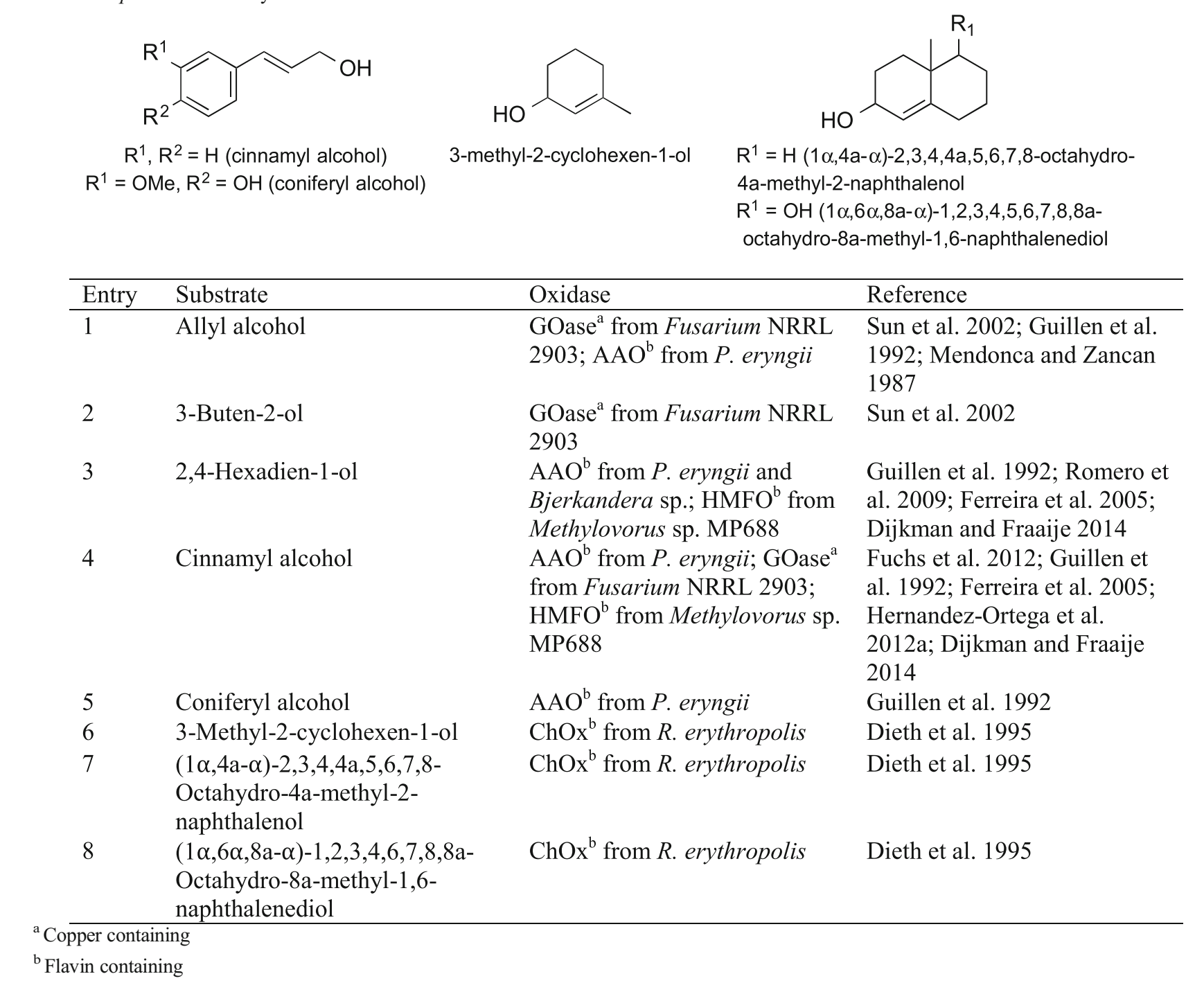




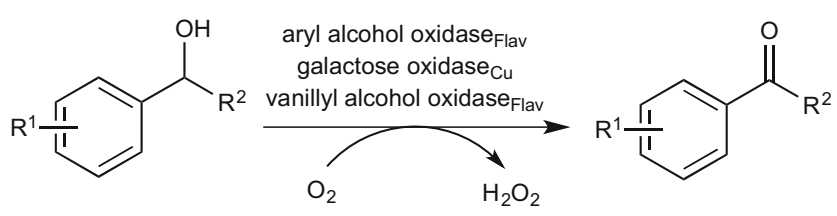

$\mathrm{R}^{1}=$ halide, hydroxy, methoxy, phenoxy, phenyl, carboxyl, nitro $\mathrm{R}^{2}=\mathrm{H}$, methyl, allyl, ethynyl, cycloalkyl

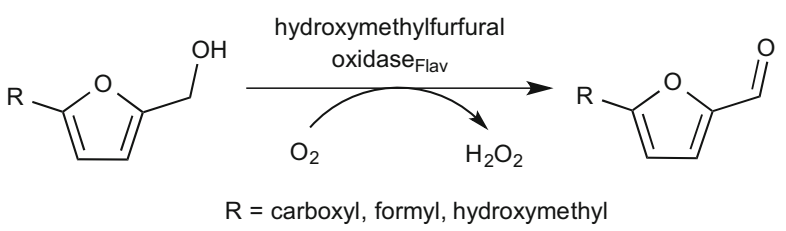

Scheme 9 Oxidation of benzylic alcohols by alcohol oxidases

substrate type, together with a recently discovered flavincontaining oxidase from Bjerkandera sp.

In the case of benzyl alcohol, two more AAOs (from A. terreus and Pleurotus ostreatus) showed activity, as well as SCAO from C. boidinii and T. aurantiacus, SAO from P. vesicularis and HMFO from Methylovorus sp. (Table 4, entry 2) (Kumar and Rapheal 2011). Although not visible on a non-chiral substrate, AAO acts in a stereoselective fashion by removing the pro- $R$ hydride as shown by deuterium experiments (Hernandez-Ortega et al. 2012b). Various substituents on the aromatic ring system are freely tolerated: Although wild-type galactose oxidase from Fusarium has a broad substrate scope for benzylic prim-alcohols, the activity was considerably increased by mutations. For instance, all regioisomers of pyridine methanol were transformed by a R330K, Q406T-mutant of galactose oxidase, which showed up to 2000-fold enhanced activity towards 2-pyridine methanol compared to the canonical D-galactose (Sun et al. 2002). Metaand para-substituted substrates (3-F, 3-Br, 3-Cl, 3- $\mathrm{NO}_{2}$, 4-F, 4$\mathrm{Cl}$, 4-Br, 4-I, 4- $\mathrm{NO}_{2}$, 4-OMe, 4-SMe, 4-Me, 4-CF 3 ) (Table 4, entries $4,6,7,17,18,20-24$ ) were converted with up to 20 fold variation of relative rates (Whittaker and Whittaker 2001).

Secondary aryl alcohols undergo kinetic resolution with partly excellent ees using an $(R)$-selective mutant of galactose oxidase from Fusarium sp. created by directed evolution (Table 4, entries 28-40) (Escalettes and Turner 2008). The same group also reported a rare example of the successful recognition of an atropisomeric pair of enantiomers possessing axial chirality (Table 4, entry 41) (Yuan et al. 2010). Furthermore, an engineered variant of HMFO was able to oxidise phenylethanol in a stereoselective fashion (Dijkman et al. 2015).

Methoxy groups (Table 4, entry 6) were accepted independently from the position on the ring with comparable activities relative to unsubstituted benzyl alcohol, whereas parasubstituted analogues reacted more than fivefold faster with aryl alcohol oxidase. Furthermore, dimethoxy benzyl alcohols (Table 4, entries 8 and 9) were converted by aryl alcohol oxidase with high activity (Hernandez-Ortega et al. 2011; Hernandez-Ortega et al. 2012a). In particular, 3,4dimethoxybenzyl alcohol (veratryl alcohol, Table 4, entry 9) was converted with $326 \%$ activity, while the 2,4-substituted pendant (Table 4 , entry 8 ) was accepted with $178 \%$ activity relative to benzyl alcohol (Guillen et al. 1992). Sterically demanding 3,4,5-trimethoxybenzyl alcohol (Table 4, entry 10) was converted slowly. Besides methoxy groups, also hydroxy groups, combinations thereof and even a meta-substituted phenoxy group were accepted (Table 4, entries 12-16). The hydroxy substrates (Table 4, entries 12 and 13) were poorly converted compared to the 3-phenoxybenzyl alcohol (Table 4, entry 16) which was well accepted (Guillen et al. 1992). Additionally, the name-giving enzyme for the VAO family, vanillyl alcohol oxidase (VAO) [EC1.1.3.38] acts on 4hydroxy-3-methoxybenzyl alcohol (vanillyl alcohol, Table 4, entry 15) (de Jong et al. 1992; Van den Heuvel et al. 1998; Fraaije et al. 1998b; Van Den Heuvel et al. 2000; Van den Heuvel et al. 2001a; Van den Heuvel et al. 2001b). While the enzyme seems to accept bulky substituents, e.g. bearing a phenoxy group, additional methoxy or especially hydroxy groups (Table 4, entries 12-16) cause unfavourable interactions in the active site. The aryl alcohol oxidase from P. eryngii also acts on 4-hydroxy-substituted $\alpha$-aryl alcohols (Table 4, entry 13) (Guillen et al. 1992). Piperonyl alcohol (1,3benzodioxole-5-methanol, Table 4, entry 11), a building block in epinephrine synthesis, was oxidised with full conversion by galactose oxidase from Fusarium sp. (Fuchs et al. 2012). A broad range of chloro- and fluoro-substituted aryl alcohols were accepted by both aryl alcohol oxidase and galactose oxidase (Table 4, entries 20 and 21) (Guillen et al. 1992; Whittaker and Whittaker 2001; Romero et al. 2009). The only exception being meta-chlorobenzyl alcohol, which was not converted at all. A substrate which is sterically demanding and well accepted by AAO is 2-naphthalene methanol (Table 4 , entry 26). It showed a relative activity of $746 \%$ compared to the monocyclic substrate analogue (Table 4, entry 2). In conclusion, the position of substituents and their polarity seem to play a crucial role in substrate acceptance. The recently characterised 5-hydroxymethylfurfural oxidase from Methylovorus sp. MP688 showed a broad substrate acceptance of various furfuryl alcohols (Table 4, entry 42), but it also showed activity on benzylic alcohols with substituents in para-position (Table 4, entries 3 and 5) and vanillyl alcohol (Table 4, entry 15) (Dijkman and Fraaije 2014). In view of the growing importance of furan derivatives, such as hydroxymethyl furfural, which can easily be obtained via double elimination of $\mathrm{H}_{2} \mathrm{O}$ from hexoses or pentoses and hence constitute a promising $\mathrm{C}$ source for organic synthesis (Schwartz et al. 2014), HMFO has a considerable potential to be used in large-scale applications. In a recent study, site-directed mutagenesis allowed to boost the activity of HMFO on 5-formyl-2-furancarboxylic acid leading to improved yields of 2,5-furandicarboxylic acid, 
Table 4 prim- and sec-Benzylic alcohols

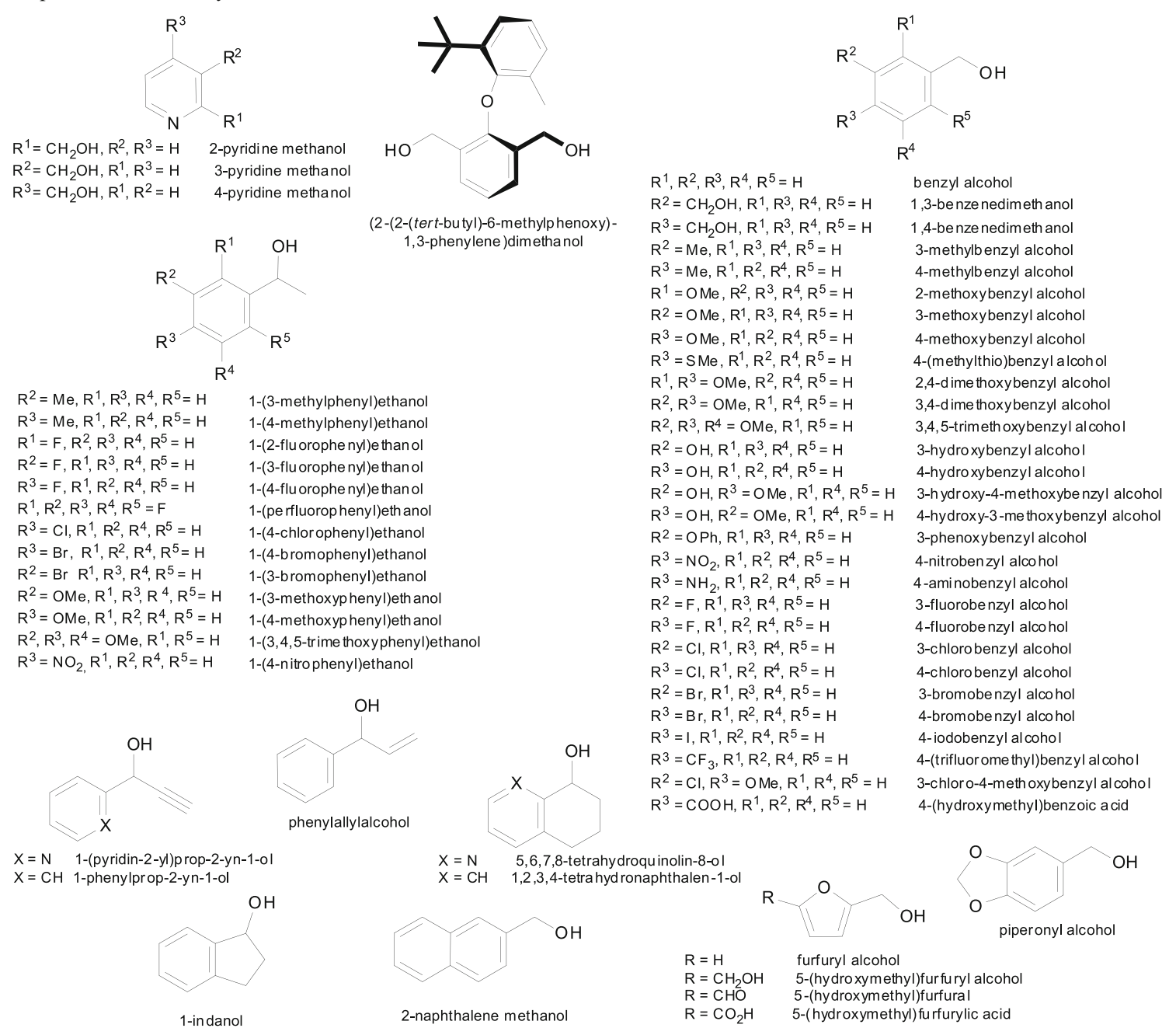

\begin{tabular}{|c|c|c|c|}
\hline Entry & Substrate & Oxidase & Reference \\
\hline 1 & $\begin{array}{l}\text { 2-Pyridine methanol } \\
\text { 3-Pyridine methanol } \\
\text { 4-Pyridine methanol }\end{array}$ & $\begin{array}{l}\text { GOase }^{\mathrm{a}} \text { from Fusarium } \\
\text { NRRL } 2903\end{array}$ & Sun et al. 2002 \\
\hline 2 & Benzyl alcohol & $\begin{array}{l}\mathrm{SCAO}^{\mathrm{b}} \text { from } C . \text { boidini; } \\
\mathrm{GOase}^{\mathrm{a}} \text { from Fusarium } \\
\mathrm{NRRL}^{2} \text { F03; } \mathrm{AAO}^{\mathrm{b}} \text { from } P . \\
\text { eryngii, A. terreus and } P . \\
\text { ostreatus and Bjerkandera } \\
\text { sp.; } \mathrm{SAO}^{\mathrm{c}} \text { from } P . \\
\text { vesicularis; } \mathrm{HMFO}^{\mathrm{b}} \text { from } \\
\text { Methylovorus } \mathrm{sp} . \mathrm{MP} 688\end{array}$ & $\begin{array}{l}\text { Fuchs et al. 2012; Ko et al. } \\
\text { 2005; Menon et al. 1995; Sakai } \\
\text { et al. 1985; Kawagoshi and } \\
\text { Fujita 1997; Guillen et al. 1992; } \\
\text { Ferreira et al. 2005; Whittaker } \\
\text { and Whittaker 2001; Hernández- } \\
\text { Ortega et al. 2012a; Kumar and } \\
\text { Rapheal 2011; Romero et al. } \\
\text { 2009; Dijkman and Fraaije } 2014\end{array}$ \\
\hline 3 & $\begin{array}{l}\text { 1,4-Benzenedimethanol } \\
\text { 1,3-Benzenedimethanol }\end{array}$ & $\begin{array}{l}\mathrm{HMFO}^{\mathrm{b}} \text { from Methylovorus } \\
\text { sp. MP688 }\end{array}$ & Dijkman and Fraaije 2014 \\
\hline
\end{tabular}

${ }^{\text {a }}$ Copper containing

${ }^{\mathrm{b}}$ Flavin containing

${ }^{\mathrm{c}} \mathrm{Fe}^{2+}$ containing 
Table 4 (continued)

\begin{tabular}{|c|c|c|c|}
\hline 4 & $\begin{array}{l}\text { 3-Methylbenzyl alcohol } \\
\text { 4-Methylbenzyl alcohol }\end{array}$ & $\begin{array}{l}\text { GOase }^{\text {a }} \text { from Fusarium } \\
\text { NRRL } 2903\end{array}$ & $\begin{array}{l}\text { Fuchs et al. 2012; Whittaker and } \\
\text { Whittaker } 2001\end{array}$ \\
\hline 5 & 4-Butylbenzyl alcohol & $\begin{array}{l}\mathrm{HMFO}^{\mathrm{b}} \text { from Methylovorus } \\
\text { sp. MP688 }\end{array}$ & Dijkman and Fraaije 2014 \\
\hline 6 & $\begin{array}{l}\text { 2-Methoxybenzyl alcohol } \\
\text { 3-Methoxybenzyl alcohol } \\
\text { 4-Methoxybenzyl alcohol }\end{array}$ & $\begin{array}{l}\text { GOase }^{\mathrm{a}} \text { from } \text { Fusarium } \\
\text { NRRL 2903; AAO }{ }^{\mathrm{b}} \text { from } P \text {. } \\
\text { eryngii and Bjerkandera sp.; } \\
\text { 4-methoxybenzyl alcohol } \\
\text { also from } P \text {. ostreatus }\end{array}$ & $\begin{array}{l}\text { Fuchs et al. 2012; Wongnate and } \\
\text { Chaiyen 2013; Guillen et al. } \\
\text { 1992; Ferreira et al. 2005; } \\
\text { Hernandez-Ortega et al. 2012a; } \\
\text { Kjellander et al. 2013; } \\
\text { Hernandez-Ortega et al. 2011; } \\
\text { Romero et al. } 2009\end{array}$ \\
\hline 7 & 4-(Methylthio)benzyl alcohol & $\begin{array}{l}\text { GOase }^{\mathrm{a}} \text { from Fusarium } \\
\text { NRRL } 2903\end{array}$ & Whittaker and Whittaker 2001 \\
\hline 8 & 2,4-Dimethoxybenzyl alcohol & $\mathrm{AAO}^{\mathrm{b}}$ from $P$. eryngii & $\begin{array}{l}\text { Wongnate and Chaiyen 2013; } \\
\text { Guillen et al. } 1992\end{array}$ \\
\hline 9 & 3,4-Dimethoxybenzyl alcohol & 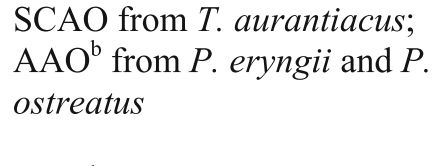 & $\begin{array}{l}\text { Ko et al. 2005; Guillen et al. } \\
\text { 1992; Hernandez-Ortega et al. } \\
\text { 2012a; Hernandez-Ortega et al. } \\
2011\end{array}$ \\
\hline 10 & 3,4,5-Trimethoxybenzyl alcohol & $\mathrm{AAO}^{\mathrm{b}}$ from $P$. eryngii & Guillen et al. 1992 \\
\hline 11 & piperonyl alcohol & $\begin{array}{l}\text { GOase }^{\mathrm{a}} \text { from Fusarium } \\
\text { NRRL } 2903\end{array}$ & Fuchs et al. 2012 \\
\hline 12 & 3-Hydroxybenzyl alcohol & $\mathrm{AAO}^{\mathrm{b}}$ from $P$. eryngii & Guillen et al. 1992 \\
\hline 13 & 4-Hydroxybenzyl alcohol & $\begin{array}{l}\mathrm{AAO}^{\mathrm{b}} \text { from } \text { P. eryngii; } \\
\text { HMFO }^{\mathrm{b}} \text { from Methylovorus } \\
\text { sp. MP688 }\end{array}$ & $\begin{array}{l}\text { Guillen et al. 1992; Dijkman and } \\
\text { Fraaije } 2014\end{array}$ \\
\hline 14 & $\begin{array}{l}\text { 3-Hydroxy-4-methoxybenzyl } \\
\text { alcohol }\end{array}$ & $\begin{array}{l}\mathrm{AAO}^{\mathrm{b}} \text { from } P . \text { eryngii and } \\
\text { Bjerkandera sp. }\end{array}$ & $\begin{array}{l}\text { Guillen et al. 1992; Ferreira et } \\
\text { al. } 2005 \text {; Romero et al. } 2009\end{array}$ \\
\hline 15 & $\begin{array}{l}\text { 4-Hydroxy-3-methoxybenzyl } \\
\text { alcohol }\end{array}$ & $\begin{array}{l}\mathrm{AAO}^{\mathrm{b}} \text { from } P . \text { eryngii and } \\
\text { Bjerkandera } \mathrm{sp} \text {.; } \mathrm{VAO}^{\mathrm{b}} \text { from } \\
\text { P. simplicissimum; } \mathrm{HMFO}^{\mathrm{b}} \\
\text { from Methylovorus } \mathrm{sp} . \\
\text { MP688 }\end{array}$ & $\begin{array}{l}\text { Guillen et al. 1992; de Jong et al. } \\
\text { 1992; Van den Heuvel et al. } \\
\text { 2001b; Romero et al. 2009; } \\
\text { Dijkman and Fraaije } 2014\end{array}$ \\
\hline 16 & 3-Phenoxybenzyl alcohol & $\mathrm{AAO}^{\mathrm{b}}$ from $P$. eryngii & Guillen et al. 1992 \\
\hline 17 & 3-Nitrobenzyl alcohol & $\begin{array}{l}\text { GOase }^{\text {a }} \text { from Fusarium } \\
\text { NRRL } 2903\end{array}$ & Whittaker and Whittaker 2001 \\
\hline 18 & 4-Nitrobenzyl alcohol & $\begin{array}{l}\text { AAO }^{\mathrm{b}} \text { from P. eryngii; } \\
\text { GOase }^{\mathrm{a}} \text { from Fusarium } \\
\text { NRRL } 2903\end{array}$ & $\begin{array}{l}\text { Guillen et al. 1992; Whittaker } \\
\text { and Whittaker } 2001\end{array}$ \\
\hline 19 & 4-Aminobenzyl alcohol & $\begin{array}{l}\text { HMFO }^{\mathrm{b}} \text { from Methylovorus } \\
\text { sp. MP688 }\end{array}$ & Dijkman and Fraaije 2014 \\
\hline 20 & $\begin{array}{l}\text { 3-Fluorobenzyl alcohol } \\
\text { 4-Fluorobenzyl alcohol }\end{array}$ & $\begin{array}{l}\text { AAO }^{\mathrm{b}} \text { from } P . \text { eryngii and } \\
\text { Bjerkandera } \text { sp.; GOase }{ }^{\mathrm{a}} \\
\text { from Fusarium NRRL } 2903\end{array}$ & $\begin{array}{l}\text { Ferreira et al. 2005; Romero et } \\
\text { al. 2009; Whittaker and } \\
\text { Whittaker } 2001\end{array}$ \\
\hline 21 & $\begin{array}{l}\text { 3-Chlorobenzyl alcohol } \\
\text { 4-Chlorobenzyl alcohol }\end{array}$ & $\begin{array}{l}\text { GOase }^{\mathrm{a}} \text { from Fusarium } \\
\text { NRRL 2903; 3-chlorobenzyl } \\
\text { alcohol also from } \mathrm{AAO}^{\mathrm{b}} \\
\text { from } P \text {. eryngii; } \mathrm{HMFO}^{\mathrm{b}} \\
\text { from Methylovorus sp. } \\
\text { MP688 }\end{array}$ & $\begin{array}{l}\text { Fuchs et al. 2012; Ferreira et al. } \\
\text { 2005; Dijkman and Fraaije } 2014\end{array}$ \\
\hline
\end{tabular}


Table 4 (continued)

\begin{tabular}{|c|c|c|c|}
\hline 22 & 3-Bromobenzyl alcohol & GOase $^{\mathrm{a}}$ from Fusarium & Whittaker and Whittaker 2001 \\
\hline & 4-Bromobenzyl alcohol & NRRL 2903 & \\
\hline 23 & 4-Iodobenzyl alcohol & $\begin{array}{l}\text { GOase }{ }^{\mathrm{a}} \text { from Fusarium } \\
\text { NRRL } 2903\end{array}$ & Whittaker and Whittaker 2001 \\
\hline 24 & $\begin{array}{l}\text { 4-(Trifluoromethyl)benzyl } \\
\text { alcohol }\end{array}$ & $\begin{array}{l}\text { GOase }^{\mathrm{a}} \text { from Fusarium } \\
\text { NRRL } 2903\end{array}$ & Whittaker and Whittaker 2001 \\
\hline 25 & $\begin{array}{l}\text { 3-Chloro-4-methoxybenzyl } \\
\text { alcohol }\end{array}$ & $\begin{array}{l}\mathrm{AAO}^{\mathrm{b}} \text { from } P . \text { eryngii and } \\
\text { Bjerkandera } \mathrm{sp} .\end{array}$ & $\begin{array}{l}\text { Ferreira et al. 2005; Romero et } \\
\text { al. } 2009\end{array}$ \\
\hline 26 & 2-Naphthalene methanol & $\mathrm{AAO}^{\mathrm{b}}$ from $P$. eryngii & $\begin{array}{l}\text { Guillen et al. 1992; Hernández- } \\
\text { Ortega et al. 2012a }\end{array}$ \\
\hline 27 & $\begin{array}{l}\text { 4-(Hydroxymethyl) } \\
\text { benzoic acid }\end{array}$ & $\mathrm{AAO}^{\mathrm{b}}$ from $P$. eryngii & Ferreira et al. 2005 \\
\hline 28 & 1-Phenylethanol & $\begin{array}{l}\text { GOase }^{\mathrm{a}} \text { from Fusarium } \\
\text { NRRL 2903; HMFO }{ }^{\mathrm{b}} \text { from } \\
\text { Methylovorus sp. MP688 }\end{array}$ & $\begin{array}{l}\text { Escalettes and Turner 2008; } \\
\text { Dijkman et al. } 2015\end{array}$ \\
\hline 29 & 1-(4-Methylphenyl)ethanol & $\begin{array}{l}\text { GOase }^{\text {a }} \text { from Fusarium } \\
\text { NRRL } 2903\end{array}$ & Escalettes and Turner 2008 \\
\hline 30 & $\begin{array}{l}\text { 1-(2-Fluorophenyl)ethanol } \\
\text { 1-(3-Fluorophenyl)ethanol } \\
\text { 1-(4-Fluorophenyl)ethanol } \\
\text { 1-(Perfluorophenyl)ethanol }\end{array}$ & $\begin{array}{l}\text { GOase }^{\text {a }} \text { from Fusarium } \\
\text { NRRL } 2903\end{array}$ & Escalettes and Turner 2008 \\
\hline 31 & 1-(4-Chlorophenyl)ethanol & $\begin{array}{l}\text { GOase }^{\text {a }} \text { from Fusarium } \\
\text { NRRL } 2903\end{array}$ & Escalettes and Turner 2008 \\
\hline 32 & $\begin{array}{l}\text { 1-(3-Bromophenyl)ethanol } \\
\text { 1-(4-Bromophenyl)ethanol }\end{array}$ & $\begin{array}{l}\text { GOase }^{\text {a }} \text { from Fusarium } \\
\text { NRRL } 2903\end{array}$ & Escalettes and Turner 2008 \\
\hline 33 & $\begin{array}{l}\text { 1-(3-Methoxyphenyl)ethanol } \\
\text { 1-(4-Methoxyphenyl)ethanol } \\
\text { 1-(3,4,5- } \\
\text { Trimethoxyphenyl)ethanol }\end{array}$ & $\begin{array}{l}\text { GOase }^{\mathrm{a}} \text { from Fusarium } \\
\text { NRRL } 2903\end{array}$ & Escalettes and Turner 2008 \\
\hline 34 & 1-(4-Nitrophenyl)ethanol & $\begin{array}{l}\text { GOase }^{\text {a }} \text { from Fusarium } \\
\text { NRRL } 2903\end{array}$ & Escalettes and Turner 2008 \\
\hline 35 & 1-Phenylallyl alcohol & $\begin{array}{l}\text { GOase a from Fusarium } \\
\text { NRRL } 2903\end{array}$ & Escalettes and Turner 2008 \\
\hline 36 & 1-Phenylprop-2-yn-1-ol & $\begin{array}{l}\text { GOase }^{\text {a }} \text { from Fusarium } \\
\text { NRRL } 2903\end{array}$ & Escalettes and Turner 2008 \\
\hline 37 & $\begin{array}{l}\text { 1,2,3,4-Tetrahydronaphthalen-1- } \\
\text { ol }\end{array}$ & $\begin{array}{l}\text { GOase }^{\text {a }} \text { from Fusarium } \\
\text { NRRL } 2903\end{array}$ & Escalettes and Turner 2008 \\
\hline 38 & 1-Indanol & $\begin{array}{l}\text { GOase }^{\text {b }} \text { from Fusarium } \\
\text { NRRL } 2903\end{array}$ & Escalettes and Turner 2008 \\
\hline 39 & $\begin{array}{l}\text { 1-(Pyridin-2-yl)ethanol } \\
\text { 1-(Pyridin-4-yl)ethanol }\end{array}$ & $\begin{array}{l}\text { GOase }^{\text {a }} \text { from Fusarium } \\
\text { NRRL } 2903\end{array}$ & Escalettes and Turner 2008 \\
\hline 40 & $5,6,7,8$-Tetrahydroquinolin-8-ol & $\begin{array}{l}\text { GOase }^{\text {a }} \text { from Fusarium } \\
\text { NRRL } 2903\end{array}$ & Escalettes and Turner 2008 \\
\hline 41 & $\begin{array}{l}\{2-[2-(\text { tert-Butyl)-6- } \\
\text { methylphenoxy]-1,3- } \\
\text { phenylene }\} \text {-dimethanol }\end{array}$ & $\begin{array}{l}\text { GOase }^{\mathrm{a}} \text { from Fusarium } \\
\text { NRRL } 2903\end{array}$ & Yuan et al. 2010 \\
\hline 42 & $\begin{array}{l}\text { Furfuryl alcohol } \\
\text { 5-(Hydroxymethyl)furfural } \\
\text { 5-Hydroxymethylfurfural } \\
\text { alcohol } \\
\text { 5-(Hydroxymethyl)furfurylic } \\
\text { acid }\end{array}$ & $\begin{array}{l}\text { HMFO }^{\text {b }} \text { from Methylovorus } \\
\text { sp. MP688 }\end{array}$ & $\begin{array}{l}\text { Dijkman and Fraaije 2014; } \\
\text { Dijkman et al. } 2015\end{array}$ \\
\hline
\end{tabular}


which is a promising monomer for polyester production from renewable resources (Dijkman et al. 2015).

\section{$\alpha$-Hydroxy acids}

Owing to the negative charge of $\alpha$-hydroxy acids at neutral $\mathrm{pH}$, the latter are oxidised by a subgroup of flavoprotein oxidases, which are specific for this type of polar substrate and furnish the corresponding $\alpha$-ketoacids (Scheme 10). On a first glimpse, this transformation appears to have little value, because it goes in hand with the destruction of a chiral centre. However, $\alpha-$ hydroxy acids are usually more easily accessible than the corresponding sensitive $\alpha$-keto-analogues, which are prone to decarboxylation; this transformation is of practical value, and in addition, racemic $\alpha$-hydroxy acids undergo kinetic resolution with a preference for the $(S)$-enantiomer (Turner 2011).

A broad range of $\alpha$-hydroxy acids were studied as substrates for FMN-depending glycolate oxidase (GlyO), L-lactate oxidase (LLO) or long-chain 2-hydroxyacid oxidase (LHAO), which belongs to the group of (S)-2-hydroxy acid oxidases (HAOX) [EC 1.1.3.15]. For GlyO, the natural substrate is glycolic acid (Table 5, entry 1) and the most prominent GlyO originates from spinach (Spinacia oleracea) (Zelitch and Ochoa 1953). The name-giving substrate can be over-oxidised to oxalic acid, although the second step is less efficient (Richardson and Tolbert 1961). Short- and mediumchain 2-hydroxy acids with up to ten carbon atoms (Table 5, entries 10 and 11), unsaturated cis- and trans-2-hydroxydec-4enoic acid (Table 5, entry 14), bulky phenyllactic acid (Table 5, entry 9) and the oxa-analogue 2-hydroxy-4pentoxybutyric acid (Table 4, entry 13) were well accepted as substrates in kinetic resolution with good to excellent ees. Furthermore, 3-chlorolactic acid (110\% rel. activity), 2hydroxybutanoic acid (120\% rel. activity), 3-indolelactic acid (18\% rel. activity) (Table 5, entries 9 and 10), 3,3,3trifluorolactic acid (11\% rel. activity) (Table 5, entry 15 ) and 2-hydroxydecanoic acid (40\% rel. activity) (Table 4, entry 12) were nicely converted relative to lactic acid (Adam et al. 1997; Adam et al. 1998; Das et al. 2009; Stenberg et al. 1995). The substrate spectrum of FMN-containing L-lactate oxidase from Aerococcus viridans and a mutant thereof encompasses also sterically demanding $\alpha$-hydroxy acids, such as para-substituted mandelic acid derivatives (Table 5, entries 5-7) and was analysed in a quantitative structure analysis (Duncan et al. 1989; Maeda-Yorita et al. 1995; Yorita et al.

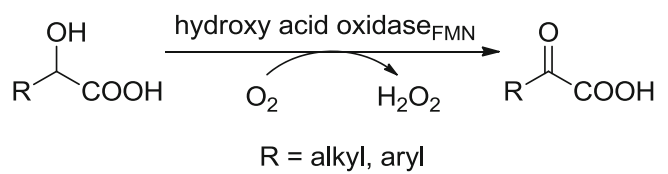

Scheme 10 Enzymatic oxidation of hydroxy acids by hydroxy acid oxidases
1997). Additionally, an enzyme originating from Pseudomonas stutzeri was used to oxidise lactic acid to pyruvate enantioselectively (Table 5 , entry 2 , Gao et al. 2009). LHAO, on the contrary, originating from mammalian sources, such as pig kidney, rat kidney or hog renal cortex, oxidises 2-hydroxy acids with a carbon chain length of at least three $\mathrm{C}$ atoms (Blanchard et al. 1946; Robinson et al. 1962). 2-Hydroxy-4methylpentanoic acid, 2-hydroxybutyric acid and also mandelic acid (Table 5, entries 8-10,12) were oxidised with moderate conversions (Urban et al. 1988).

\section{Sterols}

The bioactivity of steroids strongly depends on their substitutional pattern, which is dominated by secondary hydroxy groups in $\alpha$ - or $\beta$-positions, which upon oxidation furnish keto-steroids. This transformation can be achieved in a regio- and stereoselective fashion by alcohol oxidases. Owing to the spacious molecular framework, it is conceivable that alcohol oxidases acting on steroids have a strong preference for large substrates and are generally not ideally suited for small alcohols (Scheme 11).

Cholesterol oxidase (ChOx) [EC 1.1.3.6] found in Streptomyces hygroscopicus, Rhodococcus and Brevibacterium sterolicum is the enzyme of choice for the oxidation of the secondary alcohol function at $\mathrm{C}_{3}$, which leads to rare ketosteroids (Table 6). From a biochemical point of view, it is remarkable that cholesterol oxidases are strictly FAD containing, although they belong to two different families: Cholesterol oxidase from Streptomyces is a member of the GMC oxidase family, whereas B. sterolicum ChOx belongs to the VAO family. Remarkably, most cholesterol oxidases are bifunctional enzymes (Pollegioni et al. 1999; Gadda et al. 1997; Pollegioni et al. 2009; Vrielink and Ghisla 2009), as they not only oxidise the alcohol functionality at $\mathrm{C}_{3}$ yielding 5-cholesten-3-one but also mediate the isomerisation of the $\mathrm{C}_{5}-\mathrm{C}_{6}$ double bond of the latter into conjugation with the newly formed keto-function by assistance of an active-site glutamate residue to furnish the corresponding 4-en-3-one, as demonstrated in detail with ChOx from B. sterolicum (Kass and Sampson 1995) (Scheme 11). The enzyme exhibited a surprisingly broad substrate scope, and a variant from $R$. erythropolis even lacks enantiospecificity at the $C_{3}$ position (Dieth et al. 1995; Biellmann 2001). For the enzyme from Rhodococcus sp., moderate activities (relative to the natural substrate cholesterol) on $\beta$-sitosterol (80\% rel. activity) and stigmasterol (78 \% rel. activity) were found by Wang et al. (2008) (Table 6, entries 6 and 7). Furthermore, the enzyme was active on cholestanol, 7dehydrocholesterol and dehydroepiandrosterone (15-37\% rel. activity) (Table 6, entries 2, 4 and 8), and $5 \%$ relative activity was found on $5 \alpha$-androstane- $3 \alpha, 17 \beta$-diol (Table 6 , entry 11 ) (Labaree et al. 1997; Toyama et al. 2002; Wang et al. 2008, 
Table $5 \alpha$-Hydroxy acids

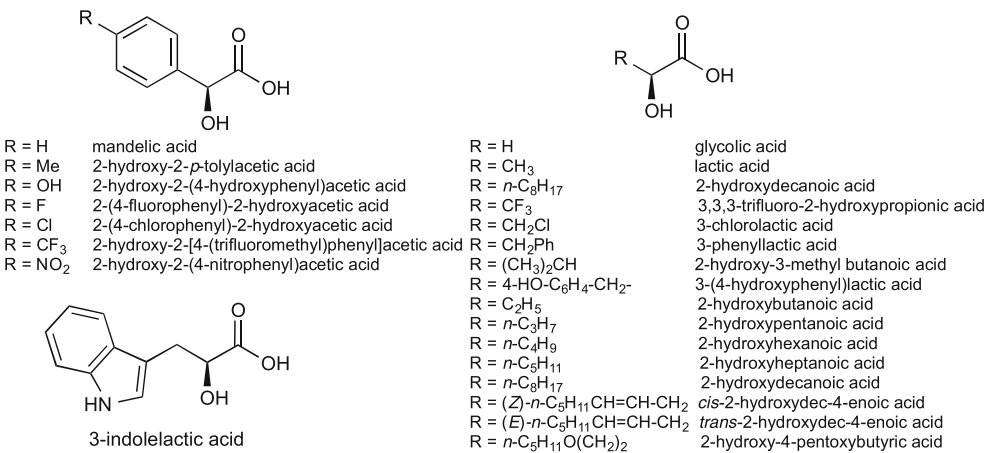

\begin{tabular}{|c|c|c|c|}
\hline Entry & Substrates & Oxidases & Reference \\
\hline 1 & Glycolic acid & $\mathrm{GlyO}^{\mathrm{b}}$ from $\mathrm{Sp}$. oleracea & $\begin{array}{l}\text { Adam et al. 1997; Stenberg } \\
\text { et al. 1995; Zelitch and } \\
\text { Ochoa 1953; Duncan et al. } \\
1989\end{array}$ \\
\hline 2 & Lactic acid & $\begin{array}{l}\mathrm{GlyO}^{\mathrm{b}} \text { from } S p . \text { oleracea; } \\
\mathrm{LLO}^{\mathrm{b}} \text { from } P . \text { stutzeri; } \\
\mathrm{LHAO}^{\mathrm{b}} \text { from } R \text {. rattus }\end{array}$ & $\begin{array}{l}\text { Adam et al. 1998; Adam et } \\
\text { al. 1997; Gao et al. 2009; } \\
\text { Stenberg et al. 1995; } \\
\text { Blanchard et al. 1946; } \\
\text { Duncan et al. 1989; } \\
\text { Maeda-Yorita et al. 1995; } \\
\text { Das et al. 2010; Urban et } \\
\text { al. } 1988\end{array}$ \\
\hline 3 & Mandelic acid & $\mathrm{GlyO}^{\mathrm{b}}$ from $S p$. oleracea & $\begin{array}{l}\text { Stenberg et al. 1995; } \\
\text { Yorita et al. 1997; Das et } \\
\text { al. 2010; Urban et al. } 1988\end{array}$ \\
\hline 4 & 2-Hydroxy-2-(4- & LLO $^{\mathrm{b}}$ from $A$. viridans & Adam et al. 1998 \\
\hline
\end{tabular}

LLO $^{\mathrm{b}}$ from A. viridans $\quad$ Yorita et al. 1997

5 2-Hydroxy-2-( $p$-tolyl)acetic acid

6 2-(4-Fluorophenyl)-2-hydroxyacetic acid

$\mathrm{LLO}^{\mathrm{b}}$ from $A$. viridans

Yorita et al. 1997

2-(4-chlorophenyl)-2-hydroxyacetic

acid

7 2-Hydroxy-2-[4-

(trifluoromethyl)phenyl]acetic acid

2-Hydroxy-2-(4-nitrophenyl)acetic acid

8 2-Hydroxy-2-phenylacetic acid 2-Methoxy-2-phenylacetic acid

9 3-Phenyllactic acid

3-Chlorolactic acid

3-Indolelactic acid

3-(4-Hydroxyphenyl)lactic acid

10 2-Hydroxybutanoic acid

2-Hydroxypentanoic acid

2-Hydroxyhexanoic acid

11 2-Hydroxyheptanoic acid

2-Hydroxydecanoic acid

12 2-Hydroxy-3-methyl butanoic acid

$\mathrm{LLO}^{\mathrm{b}}$ from $A$. viridans

Yorita et al. 1997

$\mathrm{GlyO}^{\mathrm{b}}$ from $S p$. oleracea; $\mathrm{LLO}^{\mathrm{b}}$ from $A$. viridans; LHAO $^{\mathrm{b}}$ from $R$. rattus

Adam et al. 1997; Yorita et al. 1997; Stenberg et al. 1995; Blanchard et al. 1946

$\mathrm{GlyO}^{\mathrm{b}}$ from $\mathrm{Sp}$. oleracea; 3-phenyllactic acid also from $\mathrm{LHAO}^{\mathrm{b}}$ from $R$. Das et al. 2010; Blanchard et al. 1946; Urban et al. 1988

$\mathrm{GlyO}^{\mathrm{b}}$ from $\mathrm{Sp}$. oleracea; $\mathrm{LHAO}^{\mathrm{b}}$ from $R$. rattus

Adam et al. 1997; Stenberg et al. 1995; Das et al. 2010; Blanchard et al. 1946; Urban et al. 1988

$\mathrm{GlyO}^{\mathrm{b}}$ from Sp. oleracea

Adam et al. 1997

$\mathrm{GlyO}^{\mathrm{b}}$ from Sp. oleracea; LHAO $^{\mathrm{b}}$ from $R$. rattus

Blanchard et al. 1946;

$\mathrm{GlyO}^{\mathrm{b}}$ from $\mathrm{Sp}$. oleracea

Urban et al. 1988

13 2-Hydroxy-4-pentoxybutyric acid

$\mathrm{GlyO}^{\mathrm{b}}$ from $\mathrm{Sp}$. oleracea

Adam et al. 1997

14 trans-2-Hydroxydec-4-enoic acid cis-2-Hydroxydec-4-enoic acid

15 3,3,3,-Trifluoro-2-hydroxypropionic acid

$\mathrm{GlyO}^{\mathrm{b}}$ from $\mathrm{Sp}$. oleracea

Adam et al. 1997

Das et al. 2010

\footnotetext{
${ }^{\text {a }}$ Copper containing
}

${ }^{\mathrm{b}}$ Flavin containing 


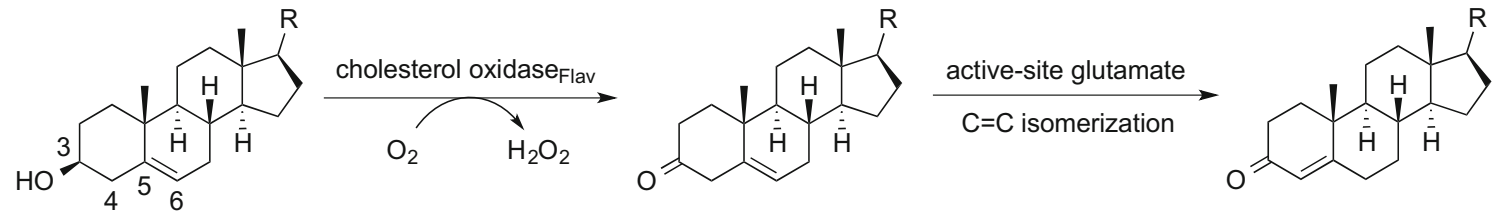

Scheme 11 Enzymatic oxidation and $\mathrm{C}=\mathrm{C}$ isomerisation of cholesterol derivatives by cholesterol oxidase

Fujishiro et al. 2002; Xiang and Sampson 2004). Moreover, cholesterol oxidase from $B$. sterolicum was employed for the oxidation of $7 \alpha$ - and $7 \beta$-hydroxycholesterol (90\% conv.) (Table 6, entry 3 ) in a chemoenzymatic multistep synthesis (Alexander and Fisher 1995).

\section{Sugar-related alcohols}

\section{Sugars}

Although sugars constitute the most abundant group of renewable compounds/materials (Straathof 2014), their polyhydroxy structure imposes several unsolved problems in view of their utility as starting materials in organic synthesis: (i) they possess only a single type of functional group - the hydroxy group, and (ii) there are too many of them with similar reactivity (Scheme 12). This causes a selectivity problem, which is usually circumvented by tedious and inefficient protection-deprotection chemistry. (iii) Furthermore, except for the anomeric carbon, the carbon framework is inaccessible to $\mathrm{C}-\mathrm{C}$ extension/modification, because the $[\mathrm{CH}-\mathrm{OH}]$ moiety cannot be directly accessed without prior activation of the hydroxy group. In this context, regioselective oxidation of $\mathrm{OH}$ groups in sugars at the expense of $\mathrm{O}_{2}$ offers an elegant method to introduce a carbonyl group, which is an ideal acceptor for $\mathrm{C}$ nucleophiles in $\mathrm{C}-\mathrm{C}$ bond forming reactions.

Due to the presence of numerous hydroxy groups, carbohydrates are usually bound in the active site of proteins via a tight hydrogen-bonding network, which is not possible for lipophilic mono-alcohols or diols. Consequently, one might surmise, that alcohol oxidases acting on lipophilic (mono) alcohols would not accept polar carbohydrates, and vice versa. However, comparison of Tables 1 and 3 shows that many sugar alcohol oxidases are also surprisingly active on small non-polar alcohols, in particular galactose oxidase and alditol oxidase.

The relative reactivity of hydroxy groups in sugars can be associated with different subgroups of alcohol oxidases, most of which possess a strong regio-preference for a specific hydroxyl group, which is exemplified on a schematic hexose (Scheme 12). With its hemiacetal structure, the anomeric $\mathrm{OH}$ is most reactive, which can be oxidised by glucose oxidase (GOX), hexose oxidase (HOX) and oligosaccharide oxidases forming the corresponding sugar lactone. Next, the terminal prim-OH is sterically least hindered among the non- activated hydroxy groups; it can be selectively oxidised by GOase to yield the aldehyde; no over-oxidation to the acid is observed in this case. Due to small steric and electronic differences, internal secondary hydroxy groups show very similar reactivities, they are oxidised by $\mathrm{P} 2 \mathrm{O}$ with mixed regioselectivities with a prevalence of $\mathrm{C}_{2}>\mathrm{C}_{3}$ yielding ketoses. $\mathrm{C}_{3}$-Oxidation products are only formed on 2-deoxy and methylated sugars.

(i) The most reactive anomeric hydroxy group in sugars can be selectively oxidised by a range of well-studied oxidases (Scheme 12): D-Glucose (Table 7, entry 1) is the natural substrate of the flavoenzyme GOX [EC 1.1.3.4], well studied from Aspergillus niger, which displayed a very narrow substrate spectrum and oxidises glucose at the $\mathrm{C}_{1}$ position (Nakamura and Ogura 1968). Furthermore, chitooligosaccharide oxidase (ChitO) [EC 1.1.3.x] from Fusarium graminearum catalyses the oxidation of $\mathrm{C}_{1}$ of D-glucose. The catalytic activity was improved by mutation (Heuts et al. 2007a), and the wild-type and mutant enzymes also accepted cellulose degradation products like cellobiose, cellotriose and cellotetraose (Table 7, entry 18). Mutants of chitooligosaccharide oxidase also accepted Dlactose and D-maltose besides the before mentioned D-glucose oligomers (Table 7, entries 9 and 10) (Heuts et al. 2007a). Variants obtained by further mutagenesis studies showed a switch in the preference for the oligosugar preference as well as improved activities on D-lactose, D-maltose and D-glucose (Ferrari et al. 2015).

Furthermore, also glucooligosaccharide oxidase (GOO) [EC 1.1.3.x] from various sources oxidised D-glucose and its oligomers at $\mathrm{C}_{1}$ (Huang et al. 2005). Lactose oxidase (LAO) [EC 1.1.3.x] from Microdochium nivale displayed a similar substrate preference. Cellobiose (Table 7, entry 18 ) with $100 \%$ relative activity was the preferred substrate, whereas di-sugars as D-maltose (84\% rel. activity) and Dlactose (52\% rel. activity) were also well accepted (Table 7, entries 9,10). Furthermore, the monosugars D-glucose (69\% rel. activity) and D-galactose (31\% rel. activity) were both oxidised at $\mathrm{C}_{1}$ (Xu et al. 2001) (Table 7, entries 1 and 2). Moreover, Pezzotti and Therisod synthesised aldonic acids starting with $\mathrm{C}_{6}$ sugars (D-galactose, D-xylose, D-mannose and 2-deoxy-D-glucose) employing glucose oxidase for the oxidation of the $\mathrm{C}_{1}$ hydroxy group (2006). HOX [EC 1.1.3.5] from Chondrus crispus is an enzyme with a fairly broad substrate scope for the oxidation of sugars at $\mathrm{C}_{1}$. Hexose oxidase accepted D-xylose, D-arabinose and D-glucose containing di-sugars, like 
Table 6 Sterols

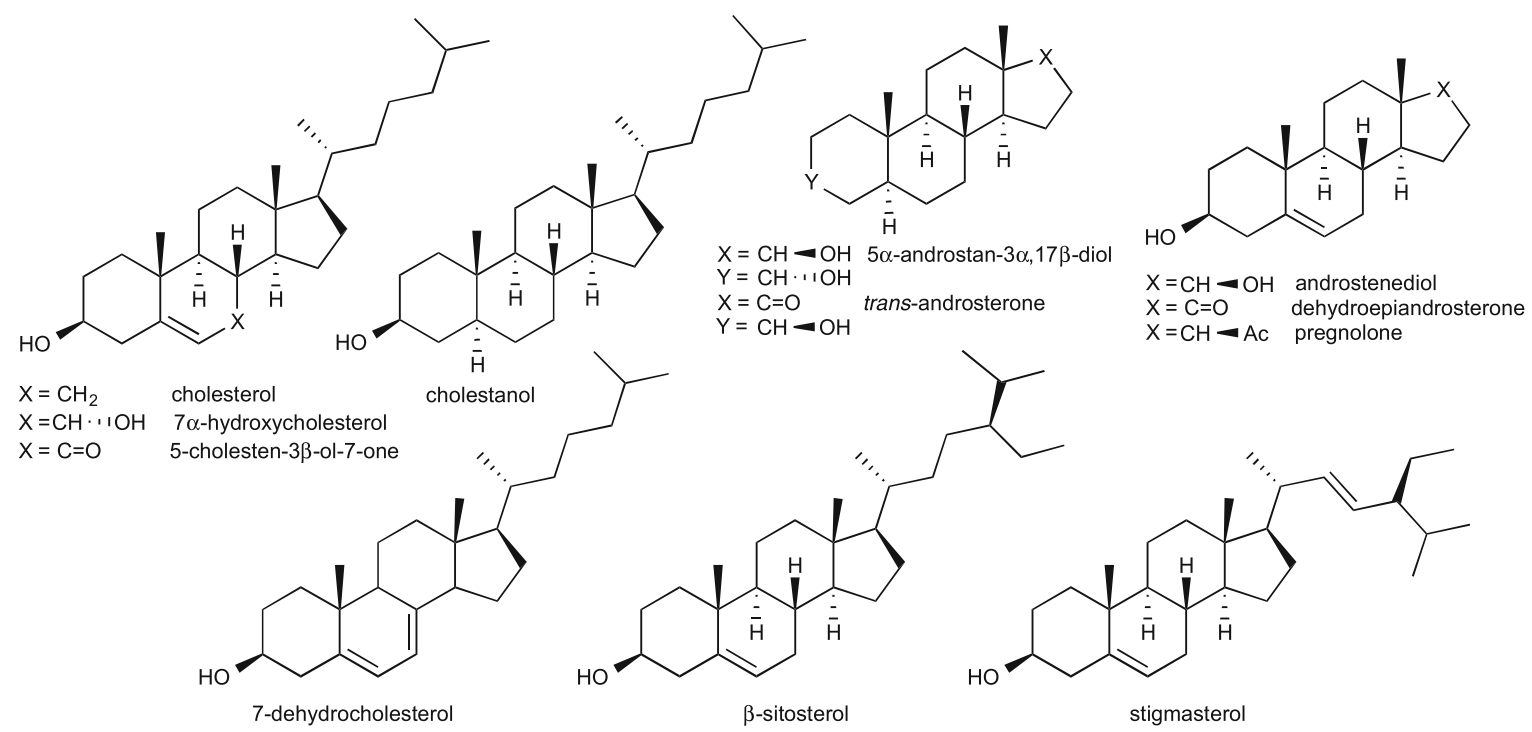

\begin{tabular}{llll}
\hline Entry & Substrate & Oxidase & Ref \\
\hline 1 & Cholesterol & ChOx ${ }^{\mathrm{b}}$ from Streptomyces $\mathrm{sp} .$, B. & D \\
& & sterolicum and Rhodococcus sp. & 2
\end{tabular}

Reference

Dieth et al. 1995; Biellmann

2001; Wang et al. 2008;

Toyama et al. 2002; Fujishiro

et al. 2002; Xiang and

Sampson 2004;

2 Cholestanol

$37 \alpha$-Hydroxycholesterol $7 \beta$-Hydroxycholesterol

4 7-Dehydrocholesterol

5 Pregnenolone

$6 \quad \beta$-Sitosterol

$7 \quad$ Stigmasterol

8 Dehydroepiandrosterone

9 trans-Androsterone

10 Dehydroisoandrosterone

$115 \alpha$-Androstan- $3 \alpha, 17 \beta$-diol

12 Androstenediol
$\mathrm{ChOx}^{\mathrm{b}}$ from $B$. sterolicum,

Streptomyces sp. and

Rhodococcus sp.

$\mathrm{ChOx}^{\mathrm{b}}$ from $B$. sterolicum

ChOx ${ }^{\mathrm{b}}$ from Rhodococcus sp.

$\mathrm{ChOx}^{\mathrm{b}}$ from $B$. sterolicum and

Streptomyces sp.

ChOx ${ }^{\mathrm{b}}$ from $B$. sterolicum,

Rhodococcus sp. and

Streptomyces sp.

$\mathrm{ChOx}^{\mathrm{b}}$ from $B$. sterolicum,

Rhodococcus sp. and

Streptomyces sp.

$\mathrm{ChOx}^{\mathrm{b}}$ from B. sterolicum and $S$. Fujishiro et al. 2002; Labaree hygroscopicus

ChOx ${ }^{\mathrm{b}}$ from $B$. sterolicum,

Rhodococcus sp. and

Streptomyces sp.

ChOx ${ }^{\mathrm{b}}$ from Streptomyces $\mathrm{sp}$.

$\mathrm{ChOx}^{\mathrm{b}}$ from Rhodococcus sp.

$\mathrm{ChOx}^{\mathrm{b}}$ from $R$. erythropolis
Wang et al. 2008; Fujishiro et al. 2002; Xiang and Sampson 2004

Alexander and Fisher 1995

Wang et al. 2008

Toyama et al. 2002; Fujishiro

et al. 2002; Xiang and

Sampson 2004

Pollegioni et al. 1999; Wang et al. 2008

Pollegioni et al. 1999; Wang et al. 2008 et al. 1997

Pollegioni et al. 1999; Wang et al. 2008; Fujishiro et al. 2002

Toyama et al. 2002

Wang et al. 2008

Biellmann 2001

\footnotetext{
${ }^{\mathrm{a}}$ Copper containing
}

${ }^{\mathrm{b}}$ Flavin containing 
D-lactose and D-cellobiose (Table 7, entries 4, 5, 10 and 18) (Poulsen and Hostrup 1998; Savary et al. 2001; Rand et al. 2006).

(ii) The sterically least hindered prim-OH group of sugars can be selectively oxidised by copper-containing galactose oxidase (Scheme 12). Relative activities were measured in relation to the reactivity of the $\mathrm{C}_{6}$-hydroxy group of D-galactose as the canonical substrate. The most prominent galactose oxidase from Fusarium converted D-galactose containing substrates D-lactose (10\% conv.), lactitol (20\% conv.), lactobionic acid and the synthetic disaccharide and laxativum D-lactulose completely (Table 7, entries 8, 10 and 17) (Siebum et al. 2006). For substrate acceptance of GOase, the axial position of the $\mathrm{C}_{4}$ position is crucial. The di-sugars D-melibiose, D-raffinose and D-stachyose were good substrates for galactose oxidase $(83 \%$ rel. activity for D-melibiose, up to $161 \%$ rel. activity for D-stachyose) (Table 7, entries 14-16) (Mendonca and Zancan 1987). For D-fructose (Table 7, entry 7), a GOase mutant from Fusarium seems to be an appropriate biocatalyst (Deacon et al. 2004). Recently, a FAD-containing hexose oxidase was discovered. The so-called Dbv29 oxidised a glycopeptide at $\mathrm{C}_{6}$ to the corresponding carboxylic acid in a twostep reaction (Li et al. 2007; Liu et al. 2011).

(iii) D-Glucose (Table 7, entry 1) was also oxidised by the flavoenzyme pyranose oxidase (P2O) [EC 1.1.3.10] (Giffhorn 2000), which was obtained from several fungi (Peniophora sp., Trametes sp., Tricholoma matsutake and Gloeophyllum sepiarium). It oxidises hydroxyl groups on the $\mathrm{C}_{2}$ position, but also oxidation at $\mathrm{C}_{3}$ can occur (Scheme 12) (Kujawa et al. 2006). The process based on $C_{2}$ oxidation of D-glucose followed by catalytic hydrogenation yielding D-fructose is known as 'Cetus process', which was also utilised for the synthesis of D-tagatose (Geigert et al. 1983; Freimund et al. 1996). D-Galactose was a rather poor substrate for pyranose oxidase from P. gigantea (Table 7, entry 2) (Freimund et al. 1998; Cook and Thygesen 2003; Bastian et al. 2005). Furthermore, the configuration on $\mathrm{C}_{4}$ played an important role in substrate acceptance. D-Allose (94\% overall yield), D-xylose (100\% overall yield) and D-mannose (only moderate rel. activity of $23 \%$ ) were all oxidised by pyranose oxidase originating from several microorganisms (Table 7, entries 3, 4 and 6) (Danneel et al. 1993; Freimund et al. 1998; Takakura and Kuwata 2003; Bannwarth et al. 2006; Machida and Nakanishi

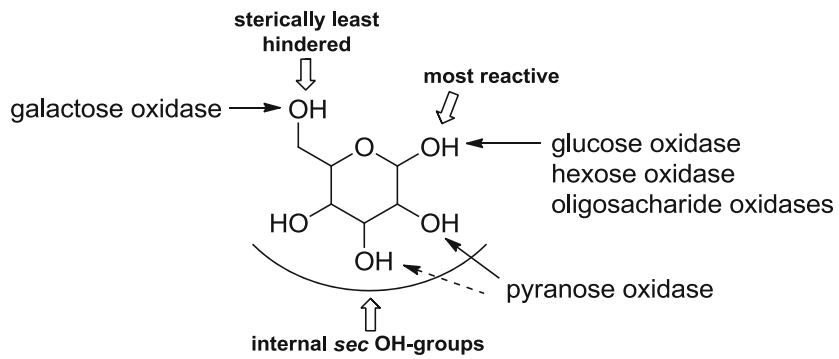

Scheme 12 Regioselectivity of alcohol oxidases on a hexose framework
1984). Pyranose oxidase accepted the di-sugars D-trehalose (54\% rel. activity), D-gentiobiose (1\% conversion) and Dmaltose (8-56 \% rel. activity) as substrates (Table 7, entries 9, 13 and 19) (Danneel et al. 1993; Freimund et al. 1998; Takakura and Kuwata 2003). Moreover, $\mathrm{P} 2 \mathrm{O}$ was used as a biocatalyst for the $\mathrm{C}_{2}$ oxidation of disaccharides to obtain 2keto-aldopyranose intermediates (Leitner et al. 2001) and the di-sugar D-sucrose (Table 7, entry 11) was fully converted by P2O in a multistep process (Seto et al. 2008).

Deoxy sugars were often employed in kinetic studies to investigate the catalytic mechanism of enzymes. 1-, 2-, 3and 6-deoxy-D-glucose and 2-deoxy-D-galactose (Table 7, entries 20-24) were used for this purpose showing full conversions. The enzymes exhibited their expected regioselectivity. For pyranose oxidase, activity was observed for 2-deoxy-Dglucose ( $52 \%$ rel. activity) for oxidation at $\mathrm{C}_{3}$ (Table 7 , entry 21). 1-Deoxy-D-glucose (Table 7, entry 20 ) was converted by pyranose oxidase $(8 \%$ rel. conversion $\mathrm{P} 2 \mathrm{O}$ from Phanerochaete gigantea, $22 \%$ from Trametes versicolor and $69 \%$ from T. matsutake). The substrate 3-deoxy-D-glucose was almost as good for pyranose oxidase as the natural one, but 6-deoxy-D-glucose showed significantly diminished relative conversion rate of $15 \%$ (Table 7, entries 22 and 23). Glucose oxidase also shows activity for 2-deoxy-D-glucose and 6-deoxy-D-glucose (Table 7, entries 21 and 23). Galactose oxidase showed $74 \%$ relative activity for 2-deoxy-D-galactose (Table 7, entry 24) (Danneel et al. 1993; Freimund et al. 1998; Takakura and Kuwata 2003; Leskovac et al. 2005; Siebum et al. 2006; Masuda-Nishimura et al. 1999).

In addition, various sugar derivatives were tested: 4-OMethylated sugars were accepted by pyranose oxidase and galactose oxidase (Schoevaart and Kieboom 2004). With pyranose oxidase, oxidation occurred at $\mathrm{C}_{3}$. Phenyl- and hexylglucosides were well accepted, but underwent a glycosyl transfer reaction forming a disaccharide (Table 7, entry 28). These bulky substrates indicate that the size of the active site is not a limiting factor. Nitro sugars were tested with pyranose oxidase, and glycosyl transfer occurred yielding a 4:1 ratio of 1-6 vs. 13 di-sugar at $C_{2}$ position in $15 \%$ overall yield. At $C_{4}$ position, a 2:1 mixture of 1-6 vs. 1-3 di-sugar was obtained in $24 \%$ yield. $\alpha$-D-Glucosyl fluoride (Table 7, entry 31 ) was a moderate substrate for pyranose oxidase from $P$. gigantea (40 \% yield, Danneel et al. 1993; Freimund et al. 1998). Pyranose oxidase also converted the unnatural L-sugar L-sorbose completely (Table 7 entry 34). Mono- and poly-fluorinated galactose analogues were oxidised by galactose oxidase (Table 7, entry 33) (Ioannou et al. 2011), and also hydroxyacetone derivatives represented excellent substrates. Dihydroxyacetone (Table 7, entry 36) was also oxidised at a fair rate by glycerol oxidase (GlycOx) from Aspergillus japonicus (59 \% rel. activity) (Uwajima and Terada 1980). Furthermore, galactose oxidase was active on guaran, a galactomannan (Table 7, entry 38) (47\% rel. activity) (Mendonca and Zancan 1987). 
Table 7 Sugars

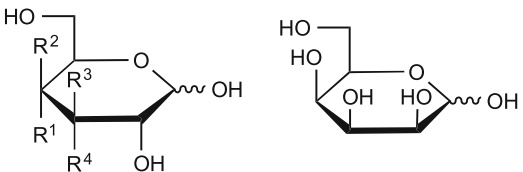

$R^{1}, R^{3}=O H, R^{2}, R^{4}=H \quad D$-glucose D-talose $R^{2}, R^{3}=O H, R^{1}, R^{4}=H \quad D$-galactose $R^{1}, R^{4}=O H, R^{2}, R^{3}=H \quad$ D-allose

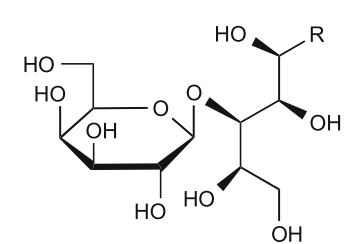

$$
\begin{array}{ll}
\mathrm{R}=\mathrm{CH}_{2} \mathrm{OH} & \text { lactitol } \\
\mathrm{R}=\mathrm{COOH} & \text { lactobionic acid }
\end{array}
$$

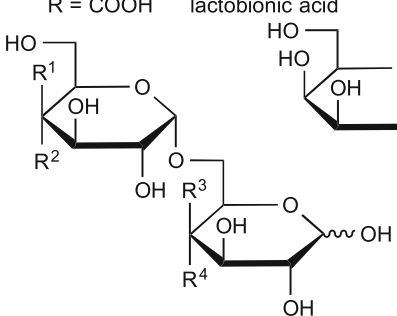

$\mathrm{R}^{1}, \mathrm{R}^{4}=\mathrm{OH}, \mathrm{R}^{2}, \mathrm{R}^{3}=\mathrm{H}$ melibiose $\mathrm{R}^{2}, \mathrm{R}^{4}=\mathrm{OH}, \mathrm{R}^{1}, \mathrm{R}^{3}=\mathrm{H}$ isomaltose $\mathrm{R}^{2}, \mathrm{R}^{3}=\mathrm{OH}, \mathrm{R}^{1}, \mathrm{R}^{4}=\mathrm{H}$ allolactose

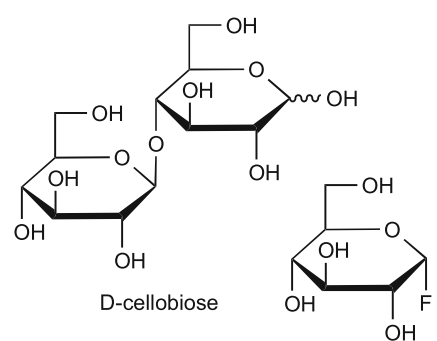

-D-glucosyl fluori

$$
\begin{array}{ll}
R^{3}, R^{4}=F, R^{1}, R^{2}=H & \text { 2,2,-difluoro-D-galactose } \\
R^{2}=F, R^{1}, R^{3}, R^{4}=H & \text { 3-fluoro-D-galactose }
\end{array}
$$
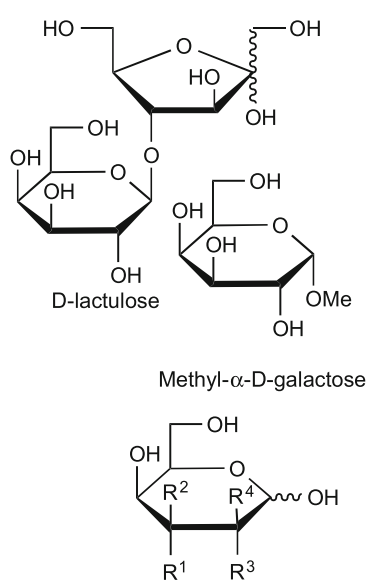

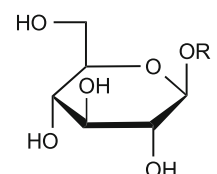

methyl- $\beta$-D-glucoside hexyl- $\beta$-D-glucoside phenyl- $\beta$-D-glucoside

$\mathrm{R}=o-\mathrm{NO}_{2}-\mathrm{C}_{6} \mathrm{H}_{4}$ 2-nitrophenyl- $\beta$-D-glucoside

$\mathrm{R}=p-\mathrm{NO}_{2}-\mathrm{C}_{6} \mathrm{H}_{4}$ 4-nitrophenyl- $\beta$-D-glucoside
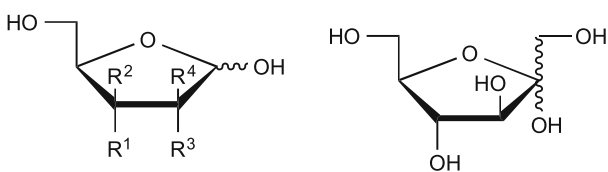

$R^{2}, R^{3}=O H, R^{1}, R^{4}=H$ D-xylose $R^{1}, R^{4}=O H, R^{2}, R^{3}=H$ D-arabinose

D-fructose ㄴo

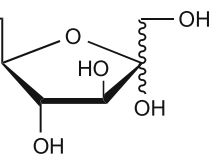

L-sorbose

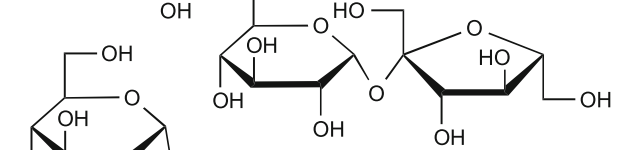

D-stachyose
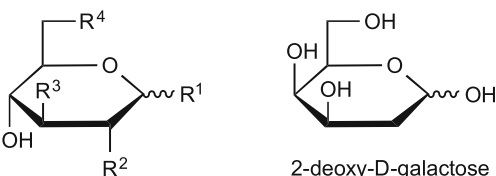

2-deoxy-D-galactose

$\mathrm{R}^{1}=\mathrm{OH}, \mathrm{R}^{2}, \mathrm{R}^{3}, \mathrm{R}^{4}=\mathrm{H}$ 1-deoxy-D-glucose $R^{2}=O H, R^{1}, R^{3}, R^{4}=H$ 2-deoxy-D-glucose $R^{3}=O H, R^{1}, R^{2}, R^{4}=H$ 3-deoxy-D-glucose $R^{4}=O H, R^{1}, R^{2}, R^{3}=H$ 6-deoxy-D-glucose

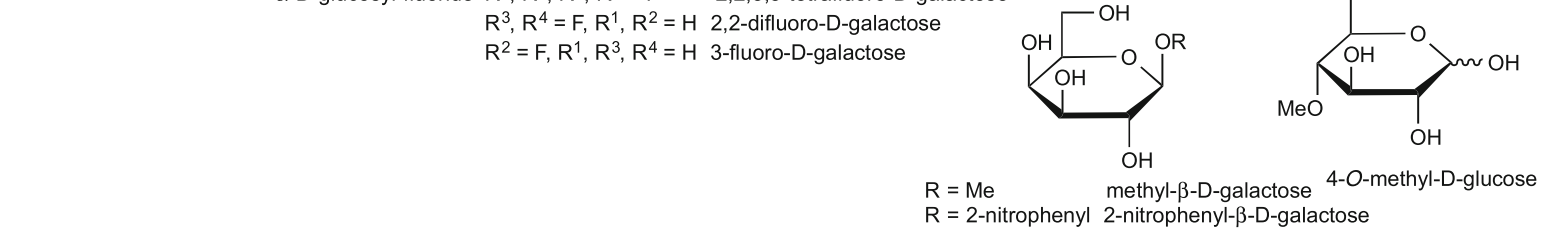

\begin{tabular}{llll}
\hline Entry & Substrate & Oxidase & Reference
\end{tabular}

\section{Mono-sugars}

1 D-Glucose

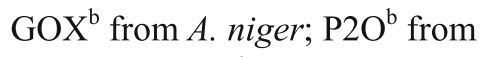
P. gigantea, T. hirsutus, $T$. versicolor, T. ochracea, $T$. matsutake, G. sepiarium and Peniophora sp.; $\mathrm{HOX}^{\mathrm{b}}$ from $C$. crispus; $\mathrm{LAO}^{\mathrm{b}}$ from M. nivale; $\mathrm{GOO}^{\mathrm{b}}$ from A. strictum; $\mathrm{ChitO}^{\mathrm{b}}$
Nakamura and Ogura 1968; Leskovac et al. 2005; Giffhorn 2000; Freimund et al. 1998; Savary et al. 2001; Bastian et al. 2005; Cook and Thygesen 2003;

Takakura and Kuwata 2003; Danneel et al. 1993; Machida

\section{${ }^{a}$ Copper containing}

${ }^{\mathrm{b}}$ Flavin containing 
Table 7 (continued)

\begin{tabular}{|c|c|c|c|}
\hline & & from $F$. graminearum & $\begin{array}{l}\text { and Nakanishi 1984; } \\
\text { Masuda-Nishimura et al. } \\
\text { 1999; Bannwarth et al. 2006; Xu } \\
\text { et al. 2001; Huang et al. 2005; } \\
\text { Heuts et al.2007a }\end{array}$ \\
\hline 2 & D-Galactose & $\begin{array}{l}\mathrm{P} 2 \mathrm{O}^{\mathrm{b}} \text { from } P . \text { gigantea, } T . \\
\text { ochracea and T. matsutake; } \\
\text { GOase }^{\mathrm{a}} \text { from Fusarium NRRL } \\
2903 ; \mathrm{HOX}^{\mathrm{b}} \text { from } C . \text { crispus; } \\
\mathrm{LAO}^{\mathrm{b}} \text { from } M . \text { nivale; } \mathrm{GOX}^{\mathrm{b}} \\
\text { from } \text { A. niger }\end{array}$ & $\begin{array}{l}\text { Siebum et al. 2006; Mendonca } \\
\text { and Zancan 1987; Freimund et } \\
\text { al. 1998; Savary et al. 2001; } \\
\text { Bastian et al. 2005; Cook and } \\
\text { Thygesen 2003; Takakura and } \\
\text { Kuwata 2003; Danneel et al. } \\
\text { 1993; Pezzotti and Therisod } 2006\end{array}$ \\
\hline 3 & D-Allose & $\begin{array}{l}\mathrm{P}^{2} \mathrm{O}^{\mathrm{b}} \text { from } P \text {. gigantea and } T \text {. } \\
\text { ochracea }\end{array}$ & $\begin{array}{l}\text { Bastian et al. 2005; Freimund et } \\
\text { al. 1998; Bannwarth et al. } 2006\end{array}$ \\
\hline 4 & D-Xylose & $\begin{array}{l}\mathrm{P}^{2} \mathrm{O}^{\mathrm{b}} \text { from } P \text {. gigantea, } T \text {. } \\
\text { versicolor, } T \text {. ochracea and } T \text {. } \\
\text { matsutake; } \mathrm{HOX}^{\mathrm{b}} \text { from } C \text {. } \\
\text { crispus; } \mathrm{GOX}^{\mathrm{b}} \text { from } A \text {. niger }\end{array}$ & $\begin{array}{l}\text { Giffhorn 2000; Bastian et al. } \\
\text { 2005; Takakura and Kuwata } \\
\text { 2003; Danneel et al. 1993; } \\
\text { Poulsen and Høstrup 1998; } \\
\text { Masuda-Nishimura et al. 1999; } \\
\text { Bannwarth et al. 2006; Pezzotti } \\
\text { and Therisod 2006 }\end{array}$ \\
\hline 5 & D-Arabinose & $\begin{array}{l}\mathrm{HOX}^{\mathrm{b}} \text { from } C . \text { crispus; } \mathrm{P}^{2} \mathrm{O}^{\mathrm{b}} \\
\text { from T. matsutake }\end{array}$ & $\begin{array}{l}\text { Cook and Thygesen 2003; } \\
\text { Takakura and Kuwata 2003; } \\
\text { Poulsen and Hostrup } 1998\end{array}$ \\
\hline 6 & D-Mannose & $\begin{array}{l}\mathrm{P}_{2} \mathrm{O}^{\mathrm{b}} \text { from } T \text {. matsutake; } \mathrm{GOX}^{\mathrm{b}} \\
\text { from } A \text {. niger }\end{array}$ & $\begin{array}{l}\text { Takakura and Kuwata 2003; } \\
\text { Pezzotti and Therisod } 2006\end{array}$ \\
\hline 7 & D-Fructose & GOase ${ }^{\mathrm{a}}$ from Fusarium sp. & Deacon et al. 2004 \\
\hline 8 & $\begin{array}{l}\text { Lactitol } \\
\text { Lactobionic acid }\end{array}$ & $\begin{array}{l}\text { GOase }^{\text {a }} \text { from Fusarium NRRL } \\
2903\end{array}$ & Siebum et al. 2006 \\
\hline \multicolumn{4}{|c|}{ Oligosugars } \\
\hline 9 & D-Maltose & $\begin{array}{l}\mathrm{P}^{2} \mathrm{O}^{\mathrm{b}} \text { from } P . \text { gigantea and } T \text {. } \\
\text { matsutake; } \mathrm{HOX}^{\mathrm{b}} \text { from } C . \\
\text { crispus; }\end{array}$ & $\begin{array}{l}\text { Cook and Thygesen 2003; } \\
\text { Takakura and Kuwata 2003; } \\
\text { Danneel et al. } 1993\end{array}$ \\
\hline 10 & D-Lactose & $\begin{array}{l}\text { GOase }{ }^{a} \text { from Fusarium NRRL } \\
\text { 2903; HOX } \text { HOm C. crispus }^{\text {from }}\end{array}$ & $\begin{array}{l}\text { Siebum et al. 2006; Mendonca } \\
\text { and Zancan 1987; Savary et al. } \\
\text { 2001; Cook and Thygesen } 2003\end{array}$ \\
\hline 11 & D-Sucrose & $\mathrm{P} 2 \mathrm{O}^{\mathrm{b}}$ from $T$. versicolor & Seto et al. 2008 \\
\hline 12 & $\begin{array}{l}\text { D-Allolactose } \\
\text { D-Isomaltose }\end{array}$ & $\mathrm{P} 2 \mathrm{O}^{\mathrm{b}}$ from $T$. multicolor & Leitner et al. 2001 \\
\hline 13 & D-Gentiobiose & $\begin{array}{l}\mathrm{P} 2 \mathrm{O}^{\mathrm{b}} \text { from } P . \text { gigantea and } T \text {. } \\
\text { multicolor }\end{array}$ & $\begin{array}{l}\text { Giffhorn 2000; Leitner et al. } \\
2001\end{array}$ \\
\hline 14 & D-Melibiose & $\begin{array}{l}\text { GOase }{ }^{\mathrm{a}} \text { from Fusarium NRRL } \\
\text { 2903; P2O }{ }^{\mathrm{b}} \text { from T. multicolor }\end{array}$ & $\begin{array}{l}\text { Siebum et al. 2006; Mendonca } \\
\text { and Zancan 1987; Leitner et al. } \\
2001\end{array}$ \\
\hline 15 & D-Raffinose & $\begin{array}{l}\text { GOase }^{\text {a }} \text { from Fusarium NRRL } \\
2903\end{array}$ & $\begin{array}{l}\text { Siebum et al. 2006; Mendonca } \\
\text { and Zancan } 1987\end{array}$ \\
\hline 16 & D-Stachyose & $\begin{array}{l}\text { GOase }^{\text {a }} \text { from Fusarium NRRL } \\
2903\end{array}$ & Mendonca and Zancan 1987 \\
\hline
\end{tabular}


Table 7 (continued)

\begin{tabular}{|c|c|c|c|}
\hline 17 & D-Lactulose & $\begin{array}{l}\text { GOase }^{a} \text { from Fusarium NRRL } \\
2903\end{array}$ & Siebum et al. 2006 \\
\hline \multirow[t]{2}{*}{18} & $\begin{array}{l}\text { D-Cellobiose } \\
\text { D-Cellotriose }\end{array}$ & $\begin{array}{l}\text { ChitO }{ }^{\mathrm{b}} \text { from } F \text {. graminearum; } \mathrm{D}- \\
\text { cellobiose also from } \mathrm{HOX}^{\mathrm{b}} \text { from }\end{array}$ & $\begin{array}{l}\text { Savary et al. 2001; Heuts et al. } \\
2007 \text { a }\end{array}$ \\
\hline & D-Cellotetraose & C. crispus & \\
\hline 19 & D-Trehalose & $\mathrm{P} 2 \mathrm{O}^{\mathrm{b}}$ from $T$. matsutake & Takakura and Kuwata 2003 \\
\hline \multicolumn{4}{|c|}{ Deoxy-sugars } \\
\hline 20 & 1-Deoxy-D-glucose & $\begin{array}{l}\mathrm{P} 2 \mathrm{O}^{\mathrm{b}} \text { from } P . \text { gigantea, } T \text {. } \\
\text { versicolor and } T . \text { matsutake }\end{array}$ & $\begin{array}{l}\text { Freimund et al. 1998; Takakura } \\
\text { and Kuwata } 2003\end{array}$ \\
\hline 21 & 2-Deoxy-D-glucose & $\begin{array}{l}\mathrm{GOX}^{\mathrm{b}} \text { from } A \text {. niger; GOase }{ }^{\mathrm{a}} \\
\text { from } \text { Fusarium NRRL } 2903 ; \\
\mathrm{P}^{\mathrm{b}} \mathrm{O}^{\mathrm{b}} \text { from } P . \text { gigantea and } T . \\
\text { ochracea; } \mathrm{GOX}^{\mathrm{b}} \text { from } A \text {. niger }\end{array}$ & $\begin{array}{l}\text { Nakamura and Ogura 1968; } \\
\text { Leskovac et al. 2005; Kujawa et } \\
\text { al. 2006; Bastian et al. 2005; } \\
\text { Freimund et al. 1998; Pezzotti } \\
\text { and Therisod } 2006\end{array}$ \\
\hline 22 & 3-Deoxy-D-glucose & $\mathrm{P} 2 \mathrm{O}^{\mathrm{b}}$ from $P$. gigantea & Freimund et al. 1998 \\
\hline 23 & 6-Deoxy-D-glucose & $\begin{array}{l}\mathrm{P}_{2} \mathrm{O}^{\mathrm{b}} \text { from } P . \text { gigantea } ; \mathrm{GOX}^{\mathrm{b}} \\
\text { from } A \text {. niger }\end{array}$ & $\begin{array}{l}\text { Leskovac et al. 2005; Freimund } \\
\text { et al. } 1998\end{array}$ \\
\hline 24 & 2-Deoxy-D-galactose & $\begin{array}{l}\text { GOase a from Fusarium NRRL } \\
2903\end{array}$ & $\begin{array}{l}\text { Siebum et al. 2006; Mendonca } \\
\text { and Zancan } 1987\end{array}$ \\
\hline \multicolumn{4}{|c|}{ Sugar derivatives } \\
\hline 25 & Methyl- $\beta$-D-glucoside & $\begin{array}{l}\mathrm{P} 2 \mathrm{O}^{\mathrm{b}} \text { from } P . \text { gigantea and } T \text {. } \\
\text { ochracea }\end{array}$ & $\begin{array}{l}\text { Kujawa et al. 2006; Freimund et } \\
\text { al. 1998; Bastian et al. 2005; } \\
\text { Danneel et al. } 1993\end{array}$ \\
\hline \multirow[t]{2}{*}{26} & Methyl- $\alpha$-D-galactoside & GOase $^{\mathrm{a}}$ from Fusarium NRRL & Mendonca and Zancan 1987; \\
\hline & Methyl- $\beta$-D-galactoside & $\begin{array}{l}2903 \text {; methyl- } \beta \text {-D-galactoside } \\
\text { also from } \mathrm{P} 2 \mathrm{O}^{\mathrm{b}} \text { from } P \text {. gigantea } \\
\text { and } T \text {. ochracea }\end{array}$ & $\begin{array}{l}\text { Siebum et al. 2006; Kujawa et } \\
\text { al. 2006; Freimund et al. } 1998\end{array}$ \\
\hline 27 & 4-O-Methyl-D-glucose & $\mathrm{GOX}^{\mathrm{b}}$ from $A$. niger & Leskovac et al. 2005 \\
\hline 28 & $\begin{array}{l}\text { Hexyl- } \beta \text {-D-glucoside } \\
\text { Phenyl- } \beta \text {-D-glucoside }\end{array}$ & $\mathrm{P} 2 \mathrm{O}^{\mathrm{b}}$ from $P$. gigantea & Freimund et al. 1998 \\
\hline 29 & $\begin{array}{l}O \text {-Nitrophenyl- } \beta \text {-D- } \\
\text { galactoside }\end{array}$ & $\begin{array}{l}\text { GOase }^{\mathrm{a}} \text { from Fusarium NRRL } \\
2903\end{array}$ & Mendonca and Zancan 1987 \\
\hline 30 & $\begin{array}{l}\text { 2-Nitrophenyl- } \beta \text {-D- } \\
\text { glucoside } \\
\text { 4-Nitrophenyl- } \beta-D- \\
\text { glucoside }\end{array}$ & $\mathrm{P}^{2} \mathrm{O}^{\mathrm{b}}$ from $P$. gigantea & Freimund et al. 1998 \\
\hline 31 & $\alpha$-D-Glucosylfluoride & $\mathrm{P} 2 \mathrm{O}^{\mathrm{b}}$ from $P$. gigantea & Freimund et al. 1998 \\
\hline 32 & $\begin{array}{l}\text { 2-Fluoro-2-deoxy-D- } \\
\text { glucose }\end{array}$ & $\mathrm{P} 2 \mathrm{O}^{\mathrm{b}}$ from $P$. gigantea & Kujawa et al. 2006 \\
\hline \multirow[t]{3}{*}{33} & 3-Fluoro-D-galactose & GOase $^{a}$ from Fusarium NRRL & Ioannou et al. 2011 \\
\hline & 2,2-Difluoro-D-galactose & 2903 & \\
\hline & $\begin{array}{l}\text { 2,2,3,3-Tetrafluoro-D- } \\
\text { galactose }\end{array}$ & & \\
\hline
\end{tabular}


Table 7 (continued)

\begin{tabular}{|c|c|c|c|}
\hline \multicolumn{4}{|c|}{ L-Sugars } \\
\hline \multicolumn{2}{|c|}{$34 \quad$ L-Sorbose } & $\begin{array}{l}\mathrm{P} 2 \mathrm{O}^{\mathrm{b}} \text { from } P \text {. gigantea, } T . \\
\text { versicolor, } T \text {. ochracea, } T \text {. } \\
\text { matsutake and Peniophora sp. }\end{array}$ & $\begin{array}{l}\text { Giffhorn 2000; Bastian et al. } \\
\text { 2005; Danneel et al. 1993; } \\
\text { Takakura and Kuwata 2003; } \\
\text { Masuda-Nishimura et al. 1999; } \\
\text { Bannwarth et al. } 2006\end{array}$ \\
\hline 35 & L-Arabinose & $\mathrm{AldO}^{\mathrm{b}}$ from $S$. coelicolor & Van Hellemond et al. 2009 \\
\hline 36 & $\begin{array}{l}1,3- \\
\text { Dihydroxypropanone }\end{array}$ & 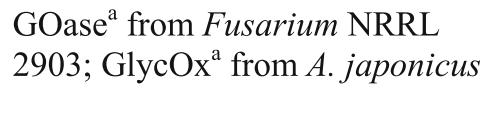 & $\begin{array}{l}\text { Siebum et al. 2006; Mendonca } \\
\text { and Zancan 1987; Uwajima and } \\
\text { Terada } 1980\end{array}$ \\
\hline 37 & $\begin{array}{l}\text { 3-Hydroxy-2-oxopropyl } \\
\text { phosphate }\end{array}$ & $\begin{array}{l}\text { GOase }^{\text {a }} \text { from Fusarium NRRL } \\
2903\end{array}$ & Franke et al. 2003 \\
\hline 38 & Guaran & $\begin{array}{l}\text { GOase }^{\text {a }} \text { from Fusarium NRRL } \\
2903\end{array}$ & Mendonca and Zancan 1987 \\
\hline 39 & $\begin{array}{l}\text { Uridine 5'-diphospho- } \alpha \text { - } \\
\text { D-galactose } \\
\text { Uridine 5'-diphospho- } N \text { - } \\
\text { acetyl- } \alpha-\mathrm{D}- \\
\text { galactosamine }\end{array}$ & $\begin{array}{l}\text { GOase }^{\text {a }} \text { from Fusarium NRRL } \\
2903\end{array}$ & $\begin{array}{l}\text { Bülter et al. 2001; Namdjou et } \\
\text { al. } 2007\end{array}$ \\
\hline
\end{tabular}

This enzyme was also applied for the oxidation of the nucleotide sugars uridine 5'-diphospho- $\alpha$-D-galactose and uridine 5'diphospho- $N$-acetyl- $\alpha$-D-galactosamine (Table 7 , entry 39 ) for subsequent biotinylation (Bülter et al. 2001; Namdjou et al. 2007).

\section{Sugar alcohols and amino sugar alcohols}

Several enzymes were reported to oxidise sugar alcohols to the corresponding aldoses, and in case of flavoprotein oxidases, aldonic acids were obtained via over-oxidation. FAD-containing alditol oxidase (AldO) [EC 1.1.3.41] has shown a broad acceptance for sugar alcohols: AldO from Streptomyces sp. and thermophilic A. cellulolyticus acted on several D- and even L-sugar alcohols (Table 8) and oxidised them to the corresponding aldoses or even further to carboxylic acids. D-Galactitol, D-xylitol, D-sorbitol, D-mannitol, L-threitol and prochiral glycerol (Table 8, entries 1-5, 9) were tested as substrates in kinetic studies (Heuts et al. 2007b; Forneris et al. 2008; Van Hellemond et al. 2009; Murooka and Yamashita 2001; Drueckhammer et al. 1991; Yamashita et al. 2000). Glycerol was oxidised to L-glyceraldehyde as a building block for a follow-up aldolase reaction in a multienzyme cascade (Franke et al. 2003). The latter is also oxidised by the $\mathrm{Cu}$-containing glycerol oxidase which exhibited excellent activity towards glycerol, which was selected as a name-giving substrate (Uwajima and Terada 1980; Uwajima et al. 1984). The building block dihydroxyacetone phosphate (DHAP), which is a popular C donor in asymmetric aldol reactions, can be obtained using flavoprotein glycerol 3-phosphate oxidase (GPO) [EC 1.1.3.21] for the oxidation of L-glycerol 3-phosphate (Table 8, entry 10) at the sec-OH (Babich et al. 2011). Furthermore, also copper-containing galactose oxidase from Fusarium exhibited a broad acceptance of sugar alcohols without acid formation (Table 8, entries 1, 2, 5, 6 and 8 ).

For the oxidation of amino sugars, $N$-acyl-D-hexosamine oxidase [EC 1.1.3.29] from Pseudomonas sp. is the enzyme of choice, although also galactose oxidase showed activities on this substrate class (Mendonca and Zancan 1987; Takahashi and Kawamura 2000). N-Acetyl-D-galactosamine (Table 8, entry 12) was converted almost as fast as the natural substrate (98-99 \% rel. activity) by $N$-acyl-D-hexosamine oxidase. It seems that (in contrast to other enzymes) the configuration of $\mathrm{C}_{4}$ is not relevant for substrate acceptance of $N$-acyl-Dhexosamine oxidase. Amino sugars without $N$-acyl function, such as D-glucosamine (26\% rel. activity) and D-galactosamine ( $81 \%$ rel. activity), were moderate substrates (Table 8 , entry 11), like $N, N^{\prime}$-diacetylchitobiose (31-49\% rel. activity) and $N$-acetylmuramic acid (44\% rel. activity) (Table 8, entry 14) with respect to the natural substrate $N$ acetyl-D-glucosamine (Horiuchi 1989; Takahashi and Kawamura 2000). The diamino sugar $N, N^{\prime}$ diacetyllactosamine and oligomers thereof were successfully oxidised by galactose oxidase (Kupper et al. 2012). Another enzyme which was found to be active on $\mathrm{C}_{1}$ of $N$-acetyl-Dglucosamine and its oligomers $N, N^{\prime}$-diacetylchitobiose, $N, N^{\prime}$, $N^{\prime \prime}$-triacetylchitotriose and $N, N^{\prime}, N^{\prime \prime}, N^{\prime \prime \prime}$-tetraacetylchitotetraose (Table 8, entry 16) is chitooligosaccharide oxidase (ChitO) (Heuts et al. 2007a) (Table 9). 
Table 8 Sugar alcohols and amino sugars<smiles></smiles><smiles>[R]CC(O)CO</smiles><smiles>OCC(O)CO</smiles><smiles>OC[C@H](O)[C@@H](O)[C@H](O)CO</smiles>
$\mathrm{R}=\mathrm{OPO}_{3}{ }^{2-}$ glycerol 3-phosphate

D-threito<smiles>[R]O[R9](=O)[C@H](O)[C@@H](O)[C@H](O)[C@H](O)CO</smiles><smiles>OC[C@H](O)[C@H](O)CO</smiles>
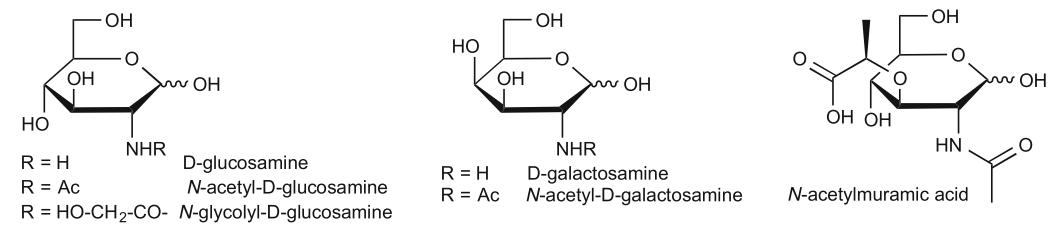

\begin{tabular}{ll}
\hline Entry & Substrate \\
\hline 1 & D-Galactitol
\end{tabular}

2

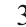

4

5

6

7

8

9

10

Amino-sugars

11 D-Glucosamine

D-Galactosamine

$N$-Acetyl-D-glucosamine $N$-Acetyl-D-galactosamine $\mathrm{N}$-Glycolyl-D-glucosamine

$14 \quad N$-Acetylmuramic acid

$N, N^{\prime}$-Diacetyllactosamine

$16 \quad N, N^{\prime}$-diacetylchitobiose $N, N^{\prime}, N^{\prime \prime}$-triacetylchitotriose $N, N^{\prime}, N^{\prime \prime}, N^{\prime \prime \prime}-$ tetraacetylchitotetraose
Oxidase Reference

AldO $^{\mathrm{b}}$ from Streptomyces sp.;

GOase $^{\text {a }}$ from Fusarium NRRL 2903

Murooka and Yamashita

2001; Drueckhammer et al.

$\mathrm{AldO}^{\mathrm{b}}$ from Streptomyces sp. and A. cellulolyticus; GOase ${ }^{\text {a }}$ from

Fusarium NRRL 2903

1991

Van Hellemond et al. 2009;

Murooka and Yamashita 2001; Winter et al. 2012;

Heuts et al. 2007b

$\mathrm{AldO}^{\mathrm{b}}$ from Streptomyces $\mathrm{sp}$. and A. cellulolyticus

etlemond et al. 2009; Murooka and Yamashita 2001; Winter et al. 2012

$\mathrm{AldO}^{\mathrm{b}}$ from Streptomyces sp. and A. cellulolyticus

$\mathrm{AldO}^{\mathrm{b}}$ from $A$. cellulolyticus; GOase $^{\text {a }}$ from Fusarium NRRL 2903; GlycOx ${ }^{\text {a }}$ from A. japonicus GOase $^{\text {a }}$ from Fusarium NRRL 2903

$\mathrm{AldO}^{\mathrm{b}}$ from Streptomyces Van Hellemond et al. 2009; Winter et al. 2012; Heuts et al. 2007b

Winter et al. 2012; Franke et al. 2003; Uwajima and

Terada 1980

Drueckhammer et al. 1991

Yamashita et al. 2000

Drueckhammer et al. 1991 2903

AldO $^{\mathrm{b}}$ from $S$. coelicolor

GPO from $S$. Thermophilus, $A$.

Murooka and Yamashita 2001; Heuts et al. 2007b viridans and Pediococcus sp.

$N$-Acyl-D-hexosamine oxidase $\mathrm{e}^{\mathrm{b}}$ from Pseudomonas sp.; Dgalactosamine also from GOase ${ }^{\mathrm{a}}$ from Fusarium NRRL 2903; Dglucosamine also from $\mathrm{GOX}^{\mathrm{b}}$ from $A$. fumigatus

$N$-Acyl-D-hexosamine oxidase $\mathrm{e}^{\mathrm{b}}$ from Pseudomonas sp.

$N$-Acyl-D-hexosamine oxidase ${ }^{\mathrm{b}}$ from Pseudomonas sp.

$N$-Acyl-D-hexosamine oxidase ${ }^{\mathrm{b}}$ from Pseudomonas sp.

GOase $^{\text {a }}$ from Fusarium NRRL 2903

$\mathrm{ChitO}^{\mathrm{b}}$ from $F$. graminearum
Babich et al. 2011

Mendonca and Zancan 1987; Horiuchi 1989; Takahashi and Kawamura 2000;

Pezzotti and Therisod 2006

Horiuchi 1989; Takahashi and Kawamura 2000

Horiuchi 1989

Horiuchi 1989

Kupper et al. 2012

Heuts et al. 2007a

${ }^{\text {a }}$ Copper containing

${ }^{\mathrm{b}}$ Flavin containing 
Table 9 Cofactor presence, substrate scope and propensity for over-oxidation of alcohol oxidases

\begin{tabular}{|c|c|c|c|}
\hline Enzyme & Cofactor & Substrate (major activities) & Over-oxidation \\
\hline Alditol oxidase (AldOx) & FAD & Primary alcohols, sugar alcohols & Yes \\
\hline Aryl alcohol oxidase (AAO) & FAD & Benzylic alcohols, allylic alcohols & Yes \\
\hline Chitooligosaccharide oxidase (ChitO) & FAD & Sugars & No \\
\hline Cholesterol oxidase (ChOx) & FAD & Sterols, allylic alcohols & No \\
\hline Choline Oxidase (CHO) & FAD & Amino alcohols & Yes \\
\hline Galactose oxidase (GOase) & $\mathrm{Cu}^{2+}$ & Benzylic alcohols, sugars & No \\
\hline Glucooligosaccharide oxidase (GOO) & FAD & Sugars & No \\
\hline Glucose oxidase (GOX) & FAD & Sugars & No \\
\hline Glycerol Oxidase (GlycOx) & $\mathrm{Cu}^{2+}$ & Sugar alcohols & No \\
\hline Glycerol 3-phosphate oxidase (GPO) & FAD & Secondary alcohols & No \\
\hline Glycolate oxidase (GlyO) & FMN & $\alpha$-Hydroxy acids & No \\
\hline Hexose oxidase (HOX) & FAD & Sugars & No \\
\hline Hydroxymethylfurfural oxidase (HMFO) & FAD & Benzylic alcohols, allylic alcohols & Yes \\
\hline (S)-2-Hydroxy acid oxidase (HAOX) & FMN & $\alpha$-Hydroxy acids & No \\
\hline Isoamyl alcohol oxidase (IAO) & FAD & Branched aliphatic alcohols & Yes \\
\hline L-lactate oxidase (LLO) & FMN & $\alpha$-Hydroxy acids & No \\
\hline Lactose oxidase (LAO) & FAD & Sugars & No \\
\hline Long-chain alcohol oxidase (LCAO) & FAD & Aliphatic alcohols & No \\
\hline Secondary alcohol oxidase (SAO) & $\mathrm{Fe}^{2+}$ & Secondary aliphatic alcohols & No \\
\hline Short-chain alcohol oxidase (SCAO) & FAD & Aliphatic alcohols & Yes \\
\hline Pyranose oxidase (P2O) & FAD & Sugars & No \\
\hline Vanillyl alcohol oxidase (VAO) & FAD & Benzylic alcohols & Yes \\
\hline
\end{tabular}

\section{Summary and outlook}

The broad substrate scope coupled with high regio- and stereoselectivity makes alcohol oxidases a fantastic tool for the oxidation of primary and secondary alcohols using molecular oxygen as an alternative to traditional chemical methods. Owing to their mechanism, copper-depending oxidases selectively yield aldehydes from primary alcohols, while overoxidation to furnish carboxylic acids may take place to a varying degree with flavin-depending oxidases. For a broad range of alcohols - non-activated prim- and sec-alcohols, activated allylic, cinnamic and benzylic alcohols, hydroxy acids, hydroxy steroids, carbohydrates and derivatives thereof-alcohol oxidases are available from various microbial sources, which are reviewed with respect to their substrate tolerance to facilitate the choice of the optimal enzyme for a given alcohol substrate.

Acknowledgments Funding by the Austrian Science Fund (FWF), within the DK Molecular Enzymology (project W9), an ErwinSchrödinger fellowship (J3466) and the Austrian BMWFW, BMVIT, SFG, Standortagentur Tirol, Government of Lower Austria and ZIT through the Austrian FFG-COMET-Funding Program is gratefully acknowledged.
Conflict of interest The authors declare that they have no competing interests.

Open Access This article is distributed under the terms of the Creative Commons Attribution 4.0 International License (http:// creativecommons.org/licenses/by/4.0/), which permits unrestricted use, distribution, and reproduction in any medium, provided you give appropriate credit to the original author(s) and the source, provide a link to the Creative Commons license, and indicate if changes were made.

\section{References}

Adam W, Lazarus M, Boss B, Saha-Möller CR, Humpf H-U, Schreier P (1997) Enzymatic resolution of chiral 2-hydroxy carboxylic acids by enantioselective oxidation with molecular oxygen catalyzed by the glycolate oxidase from spinach (Spinacia oleracea). J Org Chem 62: 7841-7843

Adam W, Lazarus M, Saha-Möller CR, Schreier P (1998) Quantitative transformation of racemic 2-hydroxy acids into ( $R$ )-2-hydroxy acids by enantioselective oxidation with glycolate oxidase and subsequent reduction of 2-keto acids with D-lactate dehydrogenase. Tetrahedron Asymmetry 9:351-355 
Alexander DL, Fisher JF (1995) A convenient synthesis of $7 \alpha$ hydroxycholest-4-en-3-one by the hydroxypropyl- 3-cyclodextrinfacilitated cholesterol oxidase oxidation of $3 \beta, 7 \alpha$-cholest- 5 -ene- 3 , 7-diol. Steroids 60:290-294

Babich L, Van Hemert LJC, Bury A, Hartog AF, Falcicchio P, Van der Oost J, Van Herk T, Wever R, Rutjes FPJT (2011) Synthesis of nonnatural carbohydrates from glycerol and aldehydes in a one-pot fourenzyme cascade reaction. Green Chem 13:2895-2900

Bannwarth M, Bastian S, Giffhorn F, Schulz GE (2006) Reaction geometry and thermostable variant of pyranose 2-oxidase from the whiterot fungus Peniophora sp. Biochemistry 45:6587-6595

Baron AJ, Stevens C, Wilmot C, Seneviratne KD, Blakeley V, Dooley DM, Phillips SEV, Knowles PF, McPherson MJ (1994) Structure and mechanism of galactose oxidase: the free radical site. J Biol Chem 269:25095-25105

Bastian S, Rekowski MJ, Witte K, Heckmann-Pohl DM, Giffhorn F (2005) Engineering of pyranose 2-oxidase from Peniophora gigantea towards improved thermostability and catalytic efficiency. Appl Microbiol Biotechnol 67:654-663

Biellmann J (2001) Resolution of alcohols by cholesterol oxidase from Rhodococcus erythropolis: lack of enantiospecificity for the steroids. Chirality 13:34-39

Blanchard M, Green DE, Nocito-Carroll V, Ratner S (1946) L-Hydroxy acid oxidase. J Biol Chem 163:137-144

Bülter T, Schumacher T, Namdjou D, Gallego R, Clausen H, Elling L, Gutie R (2001) Chemoenzymatic synthesis of biotinylated nucleotide sugars as substrates for glycosyltransferases. ChemBioChem 2: 884-894

Chaiyen P, Fraaije MW, Mattevi A (2012) The enigmatic reaction of flavins with oxygen. Trends Biochem Sci 37:373-380

Cheng Q, Liu H-T, Bombelli P, Smith A, Slabas AR (2004) Functional identification of AtFao3, a membrane bound long chain alcohol oxidase in Arabidopsis thaliana. FEBS Lett 574:62-68

Clark DS, Geresh S, DiCosimo R (1995) Enantioselective oxidation of 2methyl-1-alkanols by alcohol oxidase from methylotrophic yeasts. Bioorg Med Chem Lett 5:1383-1388

Cook MW, Thygesen HV (2003) Safety evaluation of a hexose oxidase expressed in Hansenula polymorpha. Food Chem Toxicol 41:523529

Couderc R, Baratti J (1980) Oxidation of methanol by the yeast, Pichia pastoris. purification and properties of the alcohol oxidase. Agric Biol Chem 44:2278-2289

Danneel H-J, Rössner E, Axel Z, Giffhorn F (1993) Purification and characterization of a pyranose oxidase from the basidiomycete Peniophora gigantea and chemical analyses of its reaction products. Eur J Biochem 214:795-802

Das S, Glenn JH, Subramanian M (2009) Enantioselective oxidation of 2hydroxycarboxylic acids by glycolate oxidase and catalase coexpressed in methylotrophic Pichia pastoris. Biotechnol Prog 26:607-615

De Jong E, Van Berkel WJH, Van der Zwan R, de Bont JAM (1992) Purification and characterization of vanillyl-alcohol oxidase from Penicillium simplicissimum. Eur J Biochem 208: 651-657

Deacon SE, Mahmoud K, Spooner RK, Firbank SJ, Knowles PF, Phillips SEV, McPherson MJ (2004) Enhanced fructose oxidase activity in a galactose oxidase variant. ChemBioChem 5:972-979

Dieth S, Tritsch D, Biellmann J-F (1995) Resolution of allylic alcohols by cholesterol oxidase isolated from Rhodococcus erythropolis. Tetrahedron Lett 36:2243-2246

Dijkman WP, Fraaije MW (2014) Discovery and characterization of a 5hydroxymethylfurfural oxidase from Methylovorus sp. strain MP688. Appl Environ Microbiol 80:1082-1090

Dijkman WP, de Gonzalo G, Mattevi A, Fraaije MW (2013) Flavoprotein oxidases: classification and applications. Appl Microbiol Biotechnol 97:5177-5188
Dijkman WP, Binda C, Fraaije MW, Mattevi A (2015) Structure-based enzyme tailoring of 5-hydroxymethylfurfural oxidase. ACS Catal 5: 1833-1839

Drueckhammer DG, Hennen WJ, Pederson RL, Barbas ICF, Gautheron CM, Krach T, Wong C-H (1991) Enzyme catalysis in synthetic carbohydrate chemistry. Synthesis 1991:499-525

Duncan D, Wallis JO, Torrey N, Azari MR (1989) Purification and properties of Aerococcus viridans lactate oxidase. Biochem Biophys Res Commun 164:919-926

Eirich LD, Craft DL, Steinberg L, Asif A, Eschenfeldt WH, Stols L, Donnelly MI, Wilson CR (2004) Cloning and characterization of three fatty alcohol oxidase genes from Candida tropicalis strain ATCC 20336. Appl Environ Microbiol 70:4872-4879

Escalettes F, Turner NJ (2008) Directed evolution of galactose oxidase: generation of enantioselective secondary alcohol oxidases. ChemBioChem 9:857-860

Ferrari AR, Lee M, Fraaije MW (2015) Expanding the substrate scope of chitooligosaccharide oxidase from Fusarium graminearum by structure-inspired mutagenesis. Biotechnol Bioeng 112:1074-1080

Ferreira P, Medina M, Guillen F, Martinez MJ, Van Berkel WJH, Martinez AT (2005) Spectral and catalytic properties of arylalcohol oxidase, a fungal flavoenzyme acting on polyunsaturated alcohols. Biochem J 389:731-738

Ferreira P, Hernandez-Ortega A, Herguedas B, Rencoret J, Gutierrez A, Martinez MJ, Jimenez-Barbero J, Medina M, Martinez AT (2010) Kinetic and chemical characterization of aldehyde oxidation by fungal aryl-alcohol oxidase. Biochem J 425:585-593

Fetzner S, Steiner RA (2010) Cofactor-independent oxidases and oxygenases. Appl Microbiol Biotechnol 86:791-804

Forneris F, Heuts DPHM, Delvecchio M, Rovida S, Fraaije MW, Mattevi A (2008) Structural analysis of the catalytic mechanism and stereoselectivity in Streptomyces coelicolor alditol oxidase. Biochemistry 47:978-985

Fraaije MW, Van Berkel WJH, Benen JAE, Visser J, Mattevi A (1998a) A novel oxidoreductase family sharing a conserved FAD-binding domain. Trends Biochem Sci 23:206-207

Fraaije MW, Van den Heuvel RH, Roelofs JC, Van Berkel WJH (1998b) Kinetic mechanism of vanillyl-alcohol oxidase with short-chain 4alkylphenols. Eur J Biochem 253:712-719

Franke D, Machajewski T, Hsu C-C, Wong C-H (2003) One-pot synthesis of L-fructose using coupled multienzyme systems based on rhamnulose-1-phosphate aldolase. J Org Chem 68:6828-6831

Freimund S, Huwig A, Giffhorn F, Köpper S (1996) Convenient chemoenzymatic synthesis of D-tagatose. J Carbohydr Chem 15:115-120

Freimund S, Huwig A, Giffhorn F, Köpper S (1998) Rare keto-aldoses from enzymatic oxidation: substrates and oxidation products of pyranose 2-oxidase. Chem Eur J 4:2442-2455

Fuchs M, Tauber K, Sattler J, Lechner H, Pfeffer J, Kroutil W, Faber K (2012) Amination of benzylic and cinnamic alcohols via a biocatalytic, aerobic, oxidation-transamination cascade. RSC Adv 2:62626265

Fujishiro K, Uchida H, Shimokawa K, Nakano M, Sano F, Ohta T, Kayahara N, Aisaka K, Uwajima T (2002) Purification and properties of a new Brevibacterium sterolicum cholesterol oxidase produced by E. coli MM294/pnH10. FEMS Microbiol Lett 215:243248

Gadda G (2012) Oxygen activation in flavoprotein oxidases: the importance of being positive. Biochemistry 51:2662-2669

Gadda G, Powell NLN, Menon P (2004) The trimethylammonium headgroup of choline is a major determinant for substrate binding and specificity in choline oxidase. Arch Biochem Biophys 430:264 273

Gadda G, Wels G, Pollegioni L, Zucchelli S, Ambrosius D, Pilone MS, Ghisla S (1997) Characterization of cholesterol oxidase from Streptomyces hygroscopicus and Brevibacterium sterolicum. Eur J Biochem 250:369-376 
Gao C, Qiu J, Li J, Ma C, Tang H, Xu P (2009) Enantioselective oxidation of racemic lactic acid to D-lactic acid and pyruvic acid by Pseudomonas stutzeri SDM. Bioresour Technol 100:1878-1880

Geigert J, Neidleman SL, Hirano DS (1983) Convenient, laboratory procedure for reducing D-glucosone to D-fructose. Carbohydr Res 113: $159-162$

Giffhorn F (2000) Fungal pyranose oxidases: occurrence, properties and biotechnical applications in carbohydrate chemistry. Appl Microbiol Biotechnol 54:727-740

Guengerich FP (2013) Thematic minireview series: metals in biology 2013. J Biol Chem 288:13164

Guillen F, Martinez AT, Martinez MJ (1992) Substrate specificity and properties of the aryl-alcohol oxidase from the ligninolytic fungus Pleurotus eryngii. Eur J Biochem 209:603-611

Hernandez-Ortega A, Lucas F, Ferreira P, Medina M, Guallar V, Martinez AT (2011) Modulating $\mathrm{O}_{2}$ reactivity in a fungal flavoenzyme: involvement of aryl-alcohol oxidase Phe-501 contiguous to catalytic histidine. J Biol Chem 286:41105-41114

Hernandez-Ortega A, Ferreira P, Martinez AT (2012a) Fungal arylalcohol oxidase: a peroxide-producing flavoenzyme involved in lignin degradation. Appl Microbiol Biotechnol 93:1395-1410

Hernandez-Ortega A, Ferreira P, Merino P, Medina M, Guallar V, Martinez AT (2012b) Stereoselective hydride transfer by arylalcohol oxidase, a member of the GMC superfamily. ChemBioChem 13:427-435

Heuts DPHM, Janssen DB, Fraaije MW (2007a) Changing the substrate specificity of a chitooligosaccharide oxidase from Fusarium graminearum by model-inspired site-directed mutagenesis. FEBS Lett 581:4905-4909

Heuts DPHM, Van Hellemond EW, Janssen DB, Fraaije MW (2007b) Discovery, characterization, and kinetic analysis of an alditol oxidase from Streptomyces coelicolor. J Biol Chem 282:20283-20291

Hollmann F, Arends IWCE, Buehler K, Schallmey A, Bühler B (2011) Enzyme-mediated oxidations for the chemist. Green Chem 13:226265

Horiuchi T (1989) Purification and properties of N-Acyl-D-hexosamine oxidase from Pseudomonas sp. 15-1. Agric Biol Chem 53:361-368

Huang C-H, Lai W-L, Lee M-H, Chen C-J, Vasella A, Tsai Y-C, Liaw S$\mathrm{H}$ (2005) Crystal structure of glucooligosaccharide oxidase from Acremonium strictum: a novel flavinylation of 6-S-cysteinyl, 8alpha-N1-histidyl FAD. J Biol Chem 280:38831-38838

Ikuta S, Imamura S, Misaki H, Horiuti Y (1977) Purification and characterization of choline oxidase from Arthrobacter globiformis. J Biochem 82:1741-1749

Ioannou A, Cini E, Timofte RS, Flitsch SL, Turner NJ, Linclau B (2011) Heavily fluorinated carbohydrates as enzyme substrates: oxidation of tetrafluorinated galactose by galactose oxidase. Chem Commun 47:11228-11230

Ito N, Phillips SEV, Stevens C, Ogel ZB, McPherson MJ, Keen JN, Yadav KDS, Knowles PF (1991) Novel thioether bond revealed by a $1.7 \AA$ crystal structure of galactose oxidase. Nature 350:87-90

Kass IJ, Sampson NS (1995) The isomerization catalyzed by Brevibacterium sterolicum cholesterol oxidase proceeds stereospecifically with one base. Biochem Biophys Res Commun 206:688693

Kato N, Omori Y, Tani Y, Ogata K (1976) Alcohol oxidases of Kloeckera sp. and Hansenula polymorpha. Eur J Biochem 350:341-350

Kawagoshi Y, Fujita M (1997) Purification and properties of polyvinyl alcohol oxidase with broad substrate range obtained from Pseudomonas vesicularis var. povalolyticus $\mathrm{PH}$. World J Microbiol Biotechnol 13:273-277

Kemp GD, Dickinson FM, Ratledge C (1988) Inducible long chain alcohol oxidase from alkane-grown Candida tropicalis. Appl Microbiol Biotechnol 29:370-374

Kiess M, Hecht HJ, Kalisz HM (1998) Glucose oxidase from Penicillium amagasakiense. Primary structure and comparison with other glucose-methanol-choline (GMC) oxidoreductases. Eur J Biochem 252:90-99

Kjellander M, Go K, Liljeruhm J, Boman M, Johansson G (2013) Steadystate generation of hydrogen peroxide: kinetics and stability of alcohol oxidase immobilized on nanoporous alumina. Biotechnol Lett 35:585-590

Klibanov AM, Alberti BN, Marletta MA (1982) Stereospecific oxidation of aliphatic alcohols catalyzed by galactose oxidase. Biochem Biophys Res Commun 108:804-808

Ko H-S, Yokoyama Y, Ohno N, Okadome M, Amachi S, Shinoyama H, Fujii T (2005) Purification and characterization of intracellular and extracellular, thermostable and alkali-tolerant alcohol oxidases produced by a thermophilic fungus, Thermoascus aurantiacus NBRC 31693. J Biosci Bioeng 99:348-353

Kujawa M, Ebner H, Leitner C, Hallberg BM, Prongjit M, Sucharitakul J, Ludwig R, Rudsander U, Peterbauer C, Chaiyen P, Haltrich D, Divne C (2006) Structural basis for substrate binding and regioselective oxidation of monosaccharides at $\mathrm{C} 3$ by pyranose 2-oxidase. $\mathrm{J}$ Biol Chem 281:35104-35115

Kumar AK, Goswami P (2006) Functional characterization of alcohol oxidases from Aspergillus terreus MTCC 6324. Appl Microbiol Biotechnol 72:906-911

Kumar AK, Goswami P (2008) Purification and properties of a novel broad substrate specific alcohol oxidase from Aspergillus terreus MTCC 6324. Biochim Biophys Acta 1784:1552-1559

Kumar AK, Goswami P (2009) Dissociation and reconstitution studies of a broad substrate specific multimeric alcohol oxidase protein produced by Aspergillus terreus. J Biochem 145:259-265

Kumar VV, Rapheal VS (2011) Induction and purification by three-phase partitioning of aryl alcohol oxidase (AAO) from Pleurotus ostreatus. Appl Biochem Biotechnol 163:423-432

Kupper CE, Rosencrantz RR, Henßen B, Pelantova H, Thönes S, Drozdova A, Kren V, Elling L (2012) Chemo-enzymatic modification of poly-N-acetyllactosamine (LacNAc) oligomers and N, Ndiacetyllactosamine (LacDiNAc) based on galactose oxidase treatment. J Org Chem 8:712-725

Labaree D, Hoyte RM, Hochberg RB (1997) A direct stereoselective synthesis of 7 $\beta$-hydroxytestosterone. Steroids 62:482-486

Leferink NGH, Heuts DPHM, Fraaije MW, Van Berkel WJH (2008) The growing VAO flavoprotein family. Arch Biochem Biophys 474: 292-301

Leitner C, Mayr P, Riva S, Volc J, Kulbe KD, Nidetzky B, Haltrich D (2001) Enzymatic redox isomerization of 1,6-disaccharides by pyranose oxidase and NADH-dependent aldose reductase. J Mol Catal B Enzym 11:407-414

Leskovac V, Trivić S, Wohlfahrt G, Kandrac J, Pericin D (2005) Glucose oxidase from Aspergillus niger: the mechanism of action with molecular oxygen, quinones, and one-electron acceptors. Int J Biochem Cell Biol 37:731-750

Li Y-S, Ho J-Y, Huang C-C, Lyu S-Y, Lee C-Y, Huang Y-T, Wu C-J, Chan H-C, Huang C-J, Hsu N-S, Tsai M-D, Li T-L (2007) A unique flavin mononucleotide-linked primary alcohol oxidase for glycopeptide A40926 maturation. J Am Chem Soc 129:13384-13385

Liu Y-C, Li Y-S, Lyu S-Y, Hsu L-J, Chen Y-H, Huang Y-T, Chan H-C, Huang C-J, Chen G-H, Chou C-C, Tsai M-D, Li T-L (2011) Interception of teicoplanin oxidation intermediates yields new antimicrobial scaffolds. Nat Chem Biol 7:304-309

Macheroux P, Kappes B, Ealick SE (2011) Flavogenomics-a genomic and structural view of flavin-dependent proteins. FEBS J 278:26252634

Machida Y, Nakanishi T (1984) Purification and properties of pyranose oxidase from Coriolus versicolor. Agric Biol Chem 48:2463-2470

Maeda-Yorita K, Aki K, Sagai H, Misaki H, Massey V (1995) L-Lactate oxidase and L-lactate monooxygenase: mechanistic variations on a common structural theme. Biochimie 77:631-642 
Masuda-Nishimura I, Minamihara T, Koyama Y (1999) Improvement in thermal stability and reactivity of pyranose oxidase from Coriolus versicolor by random mutagenesis. Biotechnol Lett 21:203-207

Mattevi A (2006) To be or not to be an oxidase: challenging the oxygen reactivity of flavoenzymes. Trends Biochem Sci 31:276-283

Mendonca MH, Zancan GT (1987) Purification and characterization of intracellular oxidase from Dactylium dendroides. Arch Biochem Biophys 252:507-514

Menon V, Hsieh CT, Fitzpatrick PF (1995) Substituted alcohols as mechanistic probes of alcohol oxidase. Bioorg Chem 23:42-53

Monti D, Ottolina G, Carrea G, Riva S (2011) Redox reactions catalyzed by isolated enzymes. Chem Rev 111:4111-4140

Murooka Y, Yamashita M (2001) Genetic and protein engineering of diagnostic enzymes, cholesterol oxidase and xylitol oxidase. J Biosci Bioeng 91:433-441

Nakamura BS, Ogura Y (1968) Action mechanism of glucose oxidase of Aspergillus niger. J Biochem 63:308-316

Namdjou D-J, Sauerzapfe B, Schmiedel J, Dräger G, Bernatchez S, Wakarchuk WW, Elling L (2007) Combination of UDP-Glc(NAc) 4'-epimerase and galactose oxidase in a one-pot synthesis of biotinylated nucleotide sugars. Adv Synth Catal 349:314-318

Omura K, Swern D (1978) Oxidation of alcohols by "activated" dimethyl sulfoxide. A preparative, steric and mechanistic study. Tetrahedron 34:1651-1660

Perez-Sanchez M, Müller CR, Dominguez de Maria P (2013) Multistep oxidase-lyase reactions: synthesis of optically active 2hydroxyketones by using biobased aliphatic alcohols. ChemCatChem 5:2512-2516

Pezzotti F, Therisod M (2006) Enzymatic synthesis of aldonic acids. Carbohydr Res 341:2290-2292

Pfitzner KE, Moffatt JG (1963) A new and selective oxidation of alcohols. J Am Chem Soc 85:3027-3028

Pollegioni L, Wels G, Pilone MS, Ghisla S (1999) Kinetic mechanisms of cholesterol oxidase from Streptomyces hygroscopicus and Brevibacterium sterolicum. Eur J Biochem 264:140-151

Pollegioni L, Piubelli L, Molla G (2009) Cholesterol oxidase: biotechnological applications. FEBS J 276:6857-6870

Poulsen C, Hostrup PB (1998) Purification and characterization of a hexose oxidase with excellent strengthening effects in bread. Cereal Chem J 75:51-57

Rand T, Qvist KB, Walter CP, Poulsen CH (2006) Characterization of the flavin association in hexose oxidase from Chondrus crispus. FEBS J 273:2693-2703

Richardson KE, Tolbert NE (1961) Oxidation of glyoxylic acid to oxalic acid by glycolic acid oxidase. J Biol Chem 236:1280-1284

Ridge PG, Zhang Y, Gladyshev VN (2008) Comparative genomic analyses of copper transporters and cuproproteomes reveal evolutionary dynamics of copper utilization and its link to oxygen. PLoS One 3: e1378

Robinson JC, Keay L, Molinari R, Sizer IW (1962) Cortex L- $\alpha$-hydroxy acid oxidases of hog renal cortex. J Biochem 237:2001-2010

Romero E, Ferreira P, Martínez AT, Martínez MJ (2009) New oxidase from Bjerkandera arthroconidial anamorph that oxidizes both phenolic and nonphenolic benzyl alcohols. Biochim Biophys Acta 1794:689-697

Rungsrisuriyachai K, Gadda G (2008) On the role of histidine 351 in the reaction of alcohol oxidation catalyzed by choline oxidase. Biochemistry 47:6762-6769

Sakai K, Hamada N, Watanabe Y (1985) Purification and properties of secondary alcohol oxidase with an acidic isoelectric point. Agric Biol Chem 49:817-825

Savary BJ, Hicks KB, O'Connor JV (2001) Hexose oxidase from Chondrus crispus: improved purification using perfusion chromatography. Enzym Microb Technol 29:42-51
Schoevaart R, Kieboom T (2004) Application of galactose oxidase in chemoenzymatic one-pot cascade reactions without intermediate recovery steps. Top Catal 27:3-9

Schrittwieser JH, Sattler J, Resch V, Mutti FG, Kroutil W (2011) Recent biocatalytic oxidation-reduction cascades. Curr Opin Chem Biol 15: 249-256

Schwartz TJ, O'Neill BJ, Shanks BH, Dumesic JA (2014) Bridging the chemical and biological catalysis gap: challenges and outlooks for producing sustainable chemicals. ACS Catal 4:2060-2069

Seto H, Kawakita H, Ohto K, Harada H, Inoue K (2008) Novel carbonylgroup-containing dextran synthesis by pyranose-2-oxidase and dextransucrase. Carbohydr Res 343:2417-2421

Shanmuganathan S, Natalia D, Greiner L, Domínguez de María P (2012) Oxidation-hydroxymethylation-reduction: a one-pot three-step biocatalytic synthesis of optically active $\alpha$-aryl vicinal diols. Green Chem 14:94-97

Siebum A, Van Wijk A, Schoevaart R, Kieboom T (2006) Galactose oxidase and alcohol oxidase: scope and limitations for the enzymatic synthesis of aldehydes. J Mol Catal B Enzym 41:141-145

Stenberg K, Clausen T, Lindqvist Y, Macheroux P (1995) Involvement of Tyr24 and Trp108 in substrate binding and substrate specificity of glycolate oxidase. Eur J Biochem 228:408-416

Straathof AJJ (2014) Transformation of biomass into commodity chemicals using enzymes or cells. Chem Rev 114:1871-1908

Sun L, Bulter T, Alcalde M, Petrounia IP, Arnold FH (2002) Modification of galactose oxidase to introduce glucose 6-oxidase activity. ChemBioChem 3:781-783

Takahashi S, Kawamura Y (2000) Renin inhibits N-acetyl-Dglucosamine 2 epimerase (Renin-Binding Protein). J Biochem 128:951-956

Takakura Y, Kuwata S (2003) Purification, characterization, and molecular cloning of a pyranose oxidase from the fruit body of the Basidiomycete, Tricholoma matsutake. Biosci Biotechnol Biochem 67:2598-2607

Tojo G, Fernández M (2006) Oxidation of alcohols to aldehydes and ketones: a guide to current common practice. Springer, New York

Toyama M, Yamashita M, Yoneda M, Zaborowski A, Nagato M, Ono H, Hirayama N, Murooka Y (2002) Alteration of substrate specificity of cholesterol oxidase from Streptomyces sp. by site-directed mutagenesis. Protein Eng 15:477-484

Turner NJ (2011) Enantioselective oxidation of C-O and C-N bonds using oxidases. Chem Rev 111:4073-4087

Urban P, Chirat I, Lederer F (1988) Rat kidney L-2-hydroxyacid oxidase. Structural and mechanistic comparison with flavocytochrome b2 from Baker's yeast. Biochemistry 27:7365-7371

Uwajima T, Terada O (1980) Properties of new enzyme glycerol oxidase from Aspergillus japonicus AT 008. Agric Biol Chem 44:20392045

Uwajima T, Shimizu Y, Terada O (1984) Glycerol oxidase, a novel copper hemoprotein from Aspergillus japonicus. Molecular and catalytic properties of the enzyme and its application to the analysis of serum triglycerides. J Biol Chem 259:2748-2753

Van den Heuvel RHH, Fraaije MW, Van Berkel WJH (1998) Regio- and stereospecific conversion of 4-alkylphenols by the covalent flavoprotein vanillyl-alcohol oxidase. J Bacteriol 180:5646-5651

Van Den Heuvel RHH, Fraaije MW, Ferrer M, Mattevi A, Van Berkel WJH (2000) Inversion of stereospecificity of vanillyl-alcohol oxidase. Proc Natl Acad Sci U S A 97:9455-9460

Van den Heuvel RHH, Fraaije MW, Laane C, Van Berkel WJH (2001a) Enzymatic synthesis of vanillin. J Agric Food Chem 49:2954-2958

Van den Heuvel RHH, Laane C, Van Berkel WJH (2001b) Exploring the biocatalytic potential of vanillyl-alcohol oxidase by site-directed mutagenesis. Adv Synth Catal 343:515-520

Van Hellemond EW, Vermote L, Koolen W, Sonke T, Zandvoort E, Heuts DPHM, Janssen DB, Fraaije MW (2009) Exploring the biocatalytic 
scope of alditol oxidase from Streptomyces coelicolor. Adv Synth Catal 351:1523-1530

Vennestrom PNR, Christensen CH, Pedersen S, Grunwaldt J-D, Woodley JM (2010) Next-generation catalysis for renewables: combining enzymatic with inorganic heterogeneous catalysis for bulk chemical production. ChemCatChem 2:249-258

Vrielink A, Ghisla S (2009) Cholesterol oxidase: biochemistry and structural features. FEBS J 276:6826-6843

Wang Y (1998) Catalytic galactose oxidase models: biomimetic $\mathrm{Cu}(\mathrm{II})-$ phenoxyl-radical reactivity. Science 279:537-540

Wang C, Cao Y, Sun B, Ji B, Nout MJR, Wang J, Zhao Y (2008) Preparation and some properties of cholesterol oxidase from Rhodococcus sp. R14-2. World J Microbiol Biotechnol 24:21492157

Wertz S, Studer A (2013) Nitroxide-catalyzed transition-metal-free aerobic oxidation processes. Green Chem 15:3116-3134

Whittaker JW (2003) Free radical catalysis by galactose oxidase. Chem Rev 103:2347-2363

Whittaker MM, Whittaker JW (2001) Catalytic reaction profile for alcohol oxidation by galactose oxidase. Biochemistry 40:7140-7148

Winter RT, Heuts DPHM, Rijpkema EMA, Van Bloois E, Wijma HJ, Fraaije MW (2012) Hot or not? Discovery and characterization of a thermostable alditol oxidase from Acidothermus cellulolyticus 11B. Appl Microbiol Biotechnol 95:389-403

Wongnate T, Chaiyen P (2013) The substrate oxidation mechanism of pyranose 2-oxidase and other related enzymes in the glucosemethanol-choline superfamily. FEBS J 280:3009-3027

Wongnate T, Surawatanawong P, Visitsatthawong S, Sucharitakul J, Scrutton NS, Chaiyen P (2014) Proton-coupled electron transfer and adduct configuration are important for C4a-hydroperoxyflavin formation and stabilization in a flavoenzyme. J Am Chem Soc 136: 241-253

Xiang J, Sampson NS (2004) Library screening studies to investigate substrate specificity in the reaction catalyzed by cholesterol oxidase. Protein Eng Des Sel 17:341-448

Xu F, Golightly EJ, Fuglsang CC, Schneider P, Duke KR, Lam L, Christensen S, Brown KM, Jorgensen CT, Brown SH (2001) A novel carbohydrate: acceptor oxidoreductase from Microdochium nivale. Eur J Biochem 1142:1136-1142

Yamashita N, Motoyoshi T, Nishimura A (1999) Purification and characterization of isoamyl alcohol oxidase ("Mureka"-forming enzyme). Biosci Biotechnol Biochem 63:1216-1222

Yamashita M, Omura H, Okamoto E, Furuya Y, Yabuuchi M (2000) Isolation, characterization and molecular cloning of a thermostable xylitol oxidase from Streptomyces sp. IKD472. J Biosci Bioeng 89: 350-360

Yorita K, Janko K, Aki K, Ghisla S, Palfey BA, Massey V (1997) On the reaction mechanism of L-lactate oxidase: quantitative structureactivity analysis of the reaction with para-substituted L-mandelates. Proc Natl Acad Sci U S A 94:9590-9595

Yuan B, Page A, Worrall CP, Escalettes F, Willies SC, McDouall JJW, Turner NJ, Clayden J (2010) Biocatalytic desymmetrization of an atropisomer with both an enantioselective oxidase and ketoreductases. Angew Chem Int Ed 49:7010-7013

Zelitch I, Ochoa S (1953) Oxidation and reduction of glyoxylic acids in plants: I. glycolic acid oxidase. J Biol Chem 201:707-718 